\title{
Apo E4 Alleles and Impaired Olfaction as Predictors of Alzheimer's Disease
}

\author{
Magdalena M Misiak ${ }^{1,4}$, MariaMananita S Hipolito ${ }^{2}$, Habtom W Ressom ${ }^{3}$, Thomas O Obisesan ${ }^{3}$, Kebreten F Manaye ${ }^{4}$ and Evaristus A \\ Nwulia ${ }^{1,2 *}$ \\ ${ }^{1}$ Department of Psychiatry and Behavioral Sciences, Howard University, Washington DC, USA \\ ${ }^{2}$ Department of Oncology, Lombardi Comprehensive Cancer Center, Georgetown University, Washington, DC, USA \\ ${ }^{3}$ Department of Medicine, Howard University, Washington DC, USA \\ ${ }^{4}$ Department of Physiology, Howard University, Washington DC, USA
}

\begin{abstract}
Alzheimer's disease $(A D)$ is the most common form of dementia that affects more than 5 million Americans. It is the only disease among the 10 causes of death that cannot be slowed or cured, thus raising the need for identification of early preclinical markers that could be the focus of preventative efforts. Although evidence is escalating that abnormalities in olfactory structure and function precede AD development and early cognitive impairments by one or more decades, the importance of olfaction is largely overlooked in $A D$, and such testing is not routinely performed in neurology clinics. Nevertheless, research using the olfactory model, has begun to advance our understanding of the preclinical pathophysiology of AD. Notably, an interesting series of studies is beginning to illuminate the relationship between Apolipoprotein $\mathrm{E}(\mathrm{ApoE}) \varepsilon 4$ polymorphism and olfactory dysfunction and late-onset Alzheimer's disease. In this article, we reviewed present research on the significance of ApoE and olfaction to AD, summarized current studies on the associations and mechanisms of ApoE and olfactory dysfunction, and highlighted important gaps for future work to further advance the translational application of the olfactory paradigm to early, preclinical diagnosis and treatment of $A D$.
\end{abstract}

Keywords: Apolipoprotein E; Alzheimer's disease; Olfactory bulb; Olfactory cortex; Olfactory function; Odor evoked response potentials

\section{Introduction}

\section{ApoE4 is a risk factor for $\mathrm{AD}$}

Numerous genetic studies have revealed that inheritance of ApoE4 allele increases the risk and rate of progression of late-onset Alzheimer disease, LOAD [1-3]. The human APOE gene exists as three polymorphic alleles- $-\varepsilon 2, \varepsilon 3$ and $\varepsilon 4-$ which have population frequencies of $8.4 \%, 77.9 \%$ and $13.7 \%$, respectively [4]. However, the frequency of the $\varepsilon 4$ allele is dramatically increased to $\sim 40 \%$ in patients with $\mathrm{AD}(4)$. Additionally, $65-80 \%$ of $\mathrm{AD}$ patients have at least one ApoE4 allele. Inheritance of a single ApoE $\varepsilon 4$ variant increases a person's risk of developing $\mathrm{AD}$ by a factor of three in men and four in women, and having two copies of the $\varepsilon 4$ allele increases risk by up to 15 -fold compared to persons without the $\varepsilon 4$ variant $[4,5]$. Furthermore, ApoE4 inheritance decreases the age of onset of $\mathrm{AD}[1,6-8]$.

Apolipoprotein E (ApoE) mediates lipid transport from one tissue or cell type to another $[9,10]$, thus participates in the regulation of lipid homeostasis. In peripheral tissues, ApoE is mainly produced by the liver and macrophages, and mediates cholesterol metabolism in an isoformdependent manner. ApoE4 is associated with hypercholesterolemia, which is a risk factor for atherosclerosis, coronary heart disease and stroke $[9,10]$. In the CNS, ApoE is mainly produced by astrocytes, but also can be expressed by oligodendrocytes, and ependymal layer cells $[11,12]$. Cholesterol is transported to neurons by ApoE via ApoE receptors, which are members of the low-density lipoprotein receptor (LDLR) family [13]. Increasing evidence suggests that under diverse pathophysiological conditions, CNS neurons also express ApoE, although at lower levels than astrocytes [14-17]. The cellular origin of ApoE appears to influence its effects on AD pathology $[18,19]$.

Neurofibrillary tangles and amyloid plaques, two neuropathological hallmarks of $\mathrm{AD}$, are increased in brain samples from ApoE4 carriers as compared to non-ApoE4 carriers [3,7]. Both plaques and tangles appear earlier in ApoE4 carriers as compared to non-carriers of ApoE4. In addition, $\mathrm{AD}$ patients with ApoE4 genotype showed widespread degeneration of neurons in areas of the brain related to learning and memory, compared to non-ApoE4 patients [20]. Although various hypotheses have been proposed to explain the relationship between ApoE4 and AD, the mechanism by which ApoE4 leads to AD in humans, if at all, is still unclear. A comprehensive study of the pathophysiological effect of ApoE4 on AD progression is hampered by ethical limitations to sampling of brain tissues in living persons as they progress through preclinical to clinical stages of the disease. The early involvement of the more accessible olfactory pathways by $\mathrm{AD}$ biology, therefore offers some hope that the olfactory paradigm can be applied to investigative efforts aimed at elucidating preclinical pathophysiology of disease progression in people with genetic risk of AD. This review examines the scientific premise in support of the validity of the olfactory paradigm for investigation of early and progressive $\mathrm{AD}$ in ApoE4 carriers. To provide a template for interpreting data on ApoE-related changes in olfaction in $\mathrm{AD}$, we first provide an overview of basic neurobiology of olfaction and a brief description of methods used for assessment of olfaction. This is followed by the review of extant data that examined whether the olfactory system reliably reflects the effect of apoE variations on $\mathrm{AD}$ vulnerability.

*Corresponding author: Nwulia EA, Department of Psychiatry, Howard University 2041 Georgia Ave., NW, Washington, DC 20060 USA, Tel: + 202-865-1742; E-mail: enwulia@howard.edu

Received: October 04, 2017; Accepted: October 12, 2017; Published: October 19, 2017

Citation: Misiak MM, Hipolito MMS, Ressom HW, Obisesan TO, Manaye KF, et al (2017) Apo E4 Alleles and Impaired Olfaction as Predictors of Alzheimer's Disease. Clin Exp Psychol 3: 169. doi: 10.4172/2471-2701.1000169

Copyright: (C) 2017 Misiak MM, et al. This is an open-access article distributed under the terms of the Creative Commons Attribution License, which permits unrestricted use, distribution, and reproduction in any medium, provided the original author and source are credited 
Citation: Misiak MM, Hipolito MMS, Ressom HW, Obisesan TO, Manaye KF, et al. (2017) Apo E4 Alleles and Impaired Olfaction as Predictors of Alzheimer's Disease. Clin Exp Psychol 3: 169. doi: 10.4172/2471-2701.1000169

\section{Neurobiology of olfaction}

The olfactory system enables us to perceive smell from the environment, and flavors from food. Loss of olfaction is linked to many neurodegenerative diseases, including Parkinson's disease (PD), fronto-temporal dementia (FTD) and AD [21,22]. The olfactory system begins with the olfactory mucosa, a pseudostratified columnar epithelium in the posterior region of the nasal cavity, from which odor information is projected into the olfactory bulb (OB) at the base of the brain (Figure 1). In all mammals, nasal airflow carries odorants into contact with olfactory receptors (ORs) located on the cilia of olfactory receptor neurons (ORNs) in the nasal olfactory mucosa. ORNs express only one OR type [23], and ORNs expressing the same OR innervate up to two glomeruli per OB [24]. Odorant binding with an OR triggers a G-coupled protein-mediated intracellular signaling cascade, ultimately producing an action potential $[25,26]$. The ORs possess unique chemosensory tuning properties $[27,28]$, that provide a first step at which the olfactory system can sort the limitless number of odorants it may encounter. Action potentials in the ORNs transmit odorant information into discrete zones in the $\mathrm{OB}$ whose activation is dictated by nasal airflow [29-31]. These zones in the OB form a "spatial map" of odorant information [32-35] and are modulated by local glomerular layer neurons. This type of organization is believed to be important for the most basic aspects of olfactory perception, including odor perception and discrimination [36-38]. The OB is a six-layer structure in which the sequential stages of odor information processing take place (Figure 1). The axons of ORNs form the olfactory nerve layer of the $\mathrm{OB}$ [36-38]. Secondary olfactory neurons, called mitral cells (MCs) located in the mitral cell layer of OB, and tufted cells (TCs) located in the external plexiform layer, all innervate $\mathrm{OB}$ glomeruli. Another major cell population in the $\mathrm{OB}$ are the granule cells, which are axon-less interneurons organized in patchy aggregated rows in the most central cell layer of the OB (interneuron). The apical dendrites

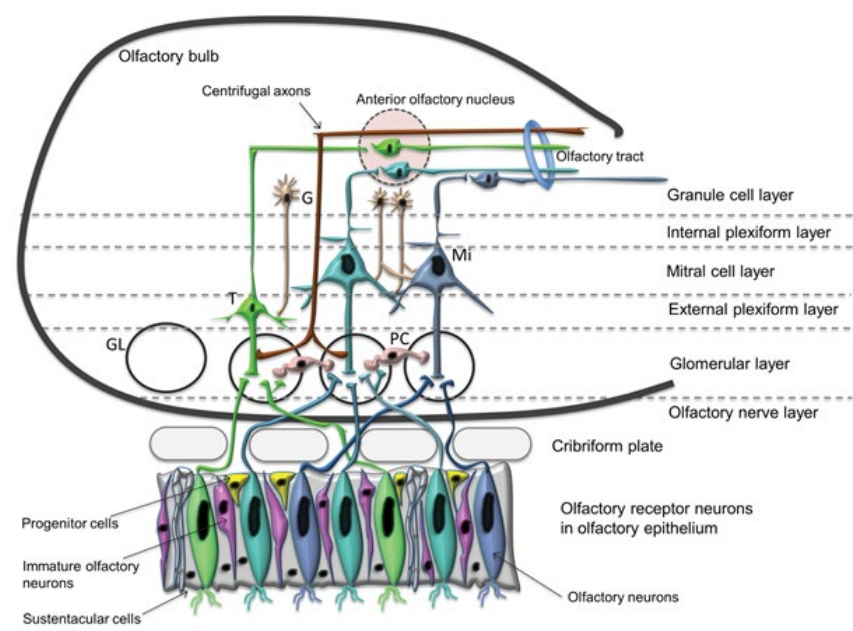

Note: G: Granule cell; M: Mitral cell; T: Tufted cell; PC: Periglomerular cells

Figure 1: Schematic diagram of the olfactory neuroepithelium (OE) and the olfactory bulb (OB). The OE consists of cells at different stages of differentiation, including the proliferating progenitor cells (yellow color), the postmitotic immature olfactory neurons (pink color) and the olfactory sensory, OSN (also known as olfactory receptor neurons, ORN). Axons from the OSN pierce through the cribriform plate at the base of the skull to enter the $O B$, where they form the olfactory nerve layer. The OB, above the OE shows the laminar organization, the major cell types and the basic neuronal circuits. Interneurons shown are the granule cells (across different layers) and the periglomerular cells in the glomerular layer (GL). Efferent neurons of the olfactory bulb are tuffed and mitral cells. of granule cells form synapsis with MCs and TCs. Granule cells also receive centrifugal input from some secondary olfactory structures and display broader odor-tuning characteristics than the upstream MCs and TCs [39]. Granule cells are mostly GABAergic and glutamatergic. In many mammalian species granule cells are constantly renewed by neurogenesis during adulthood [40-43]. The activity of MCs, TCs, and interneurons in the $\mathrm{OB}$ is subject to neuromodulation [44]. The $\mathrm{OB}$ receives dense noradrenergic projections from the locus ceruleus, cholinergic input from the horizontal limb of diagonal band of Broca, and serotoninergic afferents from the medial and dorsal raphe nuclei. Axons from MC and TC converge to form the lateral olfactory tract, whose distal projections innervate a variety of secondary olfactory structures, including the anterior olfactory nucleus $(\mathrm{AON})$, piriform cortex, olfactory tubercle, the lateral entorhinal cortex, and paraamygdaloid complex (Figure 2). These secondary olfactory structures are regarded as the primary olfactory cortex. Cells in the AON cells are highly responsive to odor stimulation [45] and they are believed to aid in intra-nostril odor localization [46]. The piriform cortex is well-known for its role in modifying the processing of odors based upon experience and learning [47-51]. Olfactory tubercle neurons are reliably activated by tasks that assess odor valence, motivated behaviors, and acquisition of rewards, suggesting important roles of the olfactory tubercle in guiding hedonic and valence-dependent responses to odors [52,53]. Neurons within these secondary olfactory structures project into tertiary olfactory structures, which include the orbitofrontal cortex, the insular cortex, and the dorsal hippocampus [54]. Of particular relevance to $\mathrm{AD}$, the entorhinal cortex innervates the hippocampus via the perforant pathway [55]. Additionally, thalamic regions receive olfactory information from several of the secondary olfactory structures, including the AON, piriform cortex, and olfactory tubercle. Olfactory information is also transmitted to the hypothalamus via the amygdaloid complex [56]. These foregoing discussions suggest that odor experience is intertwined with motivated behavior, emotion and cognition through overlap neural circuitry.

\section{Psychophysical Tasks of Olfaction}

Generally, psychophysical tasks of olfaction are based upon the presentation of odors to a test subject, followed by examination of the subject's responses to questions about some characteristics of the odorants presented. Olfactory psychophysical tasks most commonly

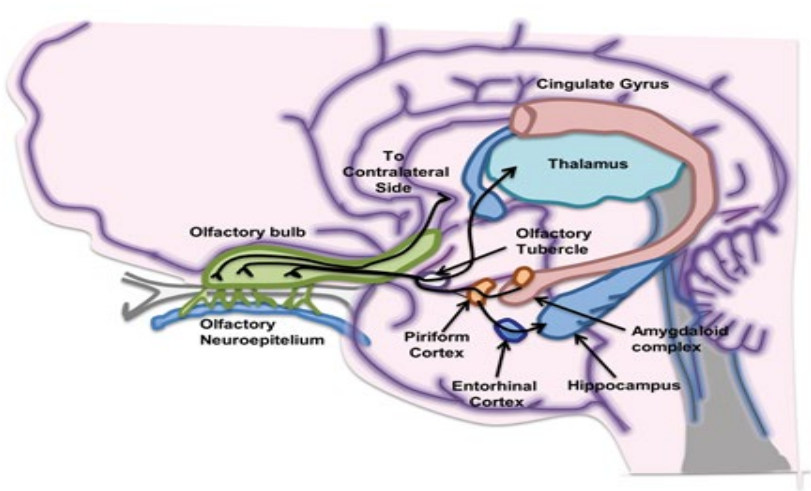

Figure 2: Simplified diagram of brain regions involved in the processing of olfactory information. The lateral olfactory tract project odor information into the primary olfactory cortex, which include the anterior olfactory nucleus (not shown), piriform cortex, olfactory tubercle, amygdaloid complex, and entorhinal cortex. From these primary olfactory cortical regions, odor information is projected to the thalamus, orbitofrontal cortex, insula cortex and hippocampus. 
measured in studies of olfaction in aging include odor identification, odor memory, odor discrimination and odor threshold sensitivity tasks [57-59]. Other methods which have been employed for clinical assessment of olfaction include surveys of subjective rating of olfactory ability and quality of life [60] and assessments of odor familiarity [61] and hedonics [62].

\section{Odor identification tasks}

During odor identification tests, odorants are presented sequentially to test subjects at suprathreshold concentrations, and subjects are required to identify each odor from a list of descriptors. This forcedchoice procedure is aimed at minimizing subjects' response bias. Odor identification tests are considered the most advanced type of test depiction of higher-order cortical functions among other psychophysical tests [63-65]. However, a major problem of odor identification test is that it correlates with the verbal abilities of the subject and has a strong cultural precondition, as not all odors are known equally well in various cultural groups. The most widely used identification tests include the 40-odorant University of Pennsylvania Smell Identification Test (UPSIT) [59] and the Sniffin' Sticks Identification Test [58]. However, several other tools are available, such as the 3-item Quick Smell Identification Test, Q-SIT [66]; the 12-item Brief Smell Identification Test, B-SIT [67]; the Smell Diskettes Olfaction Test [68]; the San Diego Odor Identification Test, (SDOIT), which consists of 8 common household odorants presented in opaque jars and includes a picture board to assist odor identification [20]; the Brief (Cross-Cultural) Smell Identification Test [67]; and the Scandinavian Odor Identification Test [69]. Additionally, odor identification can be assessed using flowdilution olfactometers, such as the T\&T Olfactometer [70].

\section{Odor discrimination tasks}

In odor discrimination procedures, different odorants are presented to subjects at suprathreshold concentrations, and subjects have to determine which of the odorants smell different [71]. This task examines the ability to distinguish between odors, not to recognize or identify them. In clinical applications discrimination tests are used in combination with identification and threshold tests. The Sniffin' Sticks Test (SST) discrimination task involves a triple-forced-choice procedure. Per triplet, two distracter pens encompass identical smells, while the respective third pen (the clue) contains a different odor. The number of correctly identified clues represents the discrimination score [71].

\section{Odor threshold tasks}

The principle behind the threshold tests is that a subject is repeatedly exposed to ascending and descending concentrations of the same odorant and is required to identify the least detectable concentration for the index odor (usually n-butanol or phenyl-ethyl-alcohol) [72]. The widespread use of the latter odorant in odor threshold applications is based on the premise that it more selectively activates olfactory receptors than the trigeminal receptors in the nose [72]. This task is normally associated with the peripheral part of the olfactory pathway [73], and can be measured by means of the T\&T olfactometer [70], the Threshold Test of the Sniffin' Sticks Extended Test [71], and the Smell Threshold Test (STT) [74].

\section{Odor memory tasks}

Perhaps due to their relative complexity, odor memory tasks were introduced more recently, compared to threshold and identification tasks. Most clinical applications of odor memory use a recognition task carried out in two stages: the acquisition and the recognition stages.
During the acquisition stage, the subject is asked to smell a small set of common household odors at intervals of 30 seconds to control for odor adaptation. This is followed by the recognition stage whereby the subject would be required to recognize the previously presented odors among distractor odors [75]. The total number of correctly recognized, percent of odors in the acquisition stage that was correctly identified, and recognition errors are outcome variables of odor memory tests commonly used in clinical studies [75,76].

\section{Neuroimaging and Physiological Measures of Olfaction}

Neuroimaging procedures, including structural and functional magnetic resonance imaging, positron emission tests (PET), single-photon emission computed tomography (SPECT), and electroencephalography (EEG), have been used in neuroscience research to characterize the neurobiology of olfactory processing. The imaging modalities provide good spatial localizations of regions relevant to olfaction, while the EEG applications reveal the sequence of neuronal activations with high temporal resolution [77]. However, due to its relative simplicity, neurobiological investigations of the relationship between ApoE and olfaction have almost exclusively employed EEG methods.

To characterize EEG patterns of olfactory perception, researchers either place electrodes intranasally to acquire odor-induced electrophysiological activity locally in the olfactory epithelium (i.e., electro-olfactography, EOG), or acquire cortical activations during odor exposure, through placement of electrodes on the scalp (i.e., odor event-related potentials, OERPs). These methods provide more objective measures of olfactory function, independent of patients' response bias. Compared to EOG, OERPs are more commonly assessed in clinical populations, and their absence is often a strong indicator of olfactory loss [78]. Odor event-related potentials (OERPs) result from the sequential activation of different brain areas, beginning from olfactory bulbs and tracts and involving the orbitofrontal and insular cortices, along with rostrum-medial regions of the temporal lobe [79]. In most OERP designs, three scalp electrodes placed along the midline - frontal $(\mathrm{Fz})$, central $(\mathrm{Cz})$, and parietal $(\mathrm{Pz})$ - regions allow for examination of relative activations of the cortical fronto-centroparietal regions by olfactory stimuli [80]. Odor event-related potentials consist of a series of positive and negative voltage deflections, which are related to a set of underlying components. Most components are referred to by a letter (N/P) indicating polarity (negative/positive), followed by a number indicating either the latency in milliseconds or the component's ordinal position in the waveform. For instance, a negative-going peak that often occurs about 100 milliseconds after a stimulus is presented is often called the N100 or N1. Typically, N1 is followed by a positive peak, known as the P200 or P2. The specified latencies for OERP components are often quite variable. For example, the P300 (P3) component may exhibit a peak anywhere between 250 $\mathrm{ms}-700 \mathrm{~ms}$ [81]. These OERP parameters are important variables in studies investigating the influence of ApoE polymorphisms in olfactory functions and in $\mathrm{AD}$.

\section{ApoE4 association with olfactory dysfunction in AD}

An overview of results from studies investigating the association between apoE polymorphisms and olfactory functions is depicted in Table 1. As shown, deficits in odor fluency, odor identification, odor recognition memory, and odor threshold sensitivity have all been associated with inheritance of the ApoE4 genotype in several studies [20,82-86]. These impairments in olfactory psychophysics are observed early in the course of $\mathrm{AD}$, even before the onset of clinical dementia 
Citation: Misiak MM, Hipolito MMS, Ressom HW, Obisesan TO, Manaye KF, et al. (2017) Apo E4 Alleles and Impaired Olfaction as Predictors of Alzheimer's Disease. Clin Exp Psychol 3: 169. doi: 10.4172/2471-2701.1000169

Page 4 of 13

\begin{tabular}{|c|c|c|c|c|c|}
\hline Study & Sample (n) & Age (years) & Measure & Findings & Other notes \\
\hline Wang et al. [85] & $\begin{array}{l}\mathrm{E} 4+(25) \\
\mathrm{E} 4-(33)\end{array}$ & $\begin{aligned} E 4+=70.70 & \pm 7.36 ; E 4-=71.67 \\
& \pm 6.28\end{aligned}$ & $\begin{array}{l}\text { Cross-cultural Smell } \\
\text { Identification Test } \\
\text { (CC-SIT) }\end{array}$ & $\begin{array}{l}\text { CC-SIT=9.11 } \pm 1.37 \text { in E4- and CC- } \\
\text { SIT }=7.34 \pm 1.43 \text { in E4+; } p<0.01\end{array}$ & $\begin{array}{c}\downarrow \text { smell identification in ApoE4 } \\
\text { group }\end{array}$ \\
\hline $\begin{array}{l}\text { Kowalewski and } \\
\text { Murphy [98] }\end{array}$ & $\begin{array}{l}\mathrm{E} 4+(10) \\
\mathrm{E} 4-(10)\end{array}$ & $69.3(4.2)$ & $\begin{array}{l}\text { Olfactory- } \\
\text { visual semantic } \\
\text { congruency task } \\
\text { to investigate } \\
\text { cross-modal odor } \\
\text { identification } \\
\text { disturbances }\end{array}$ & $\begin{array}{c}\text { Significant differences in OERPs } \\
\text { between E4+, and E4-. } \\
\text { Significant main effect for congruency } \\
(F(1,16)=27.08, p<0.001) \text {. } \\
\text { Significant 4-way interaction between } \\
\text { LR, DV, congruency and ApoE4 ( } F \\
(1,16)=9.41, p=0.007) \text {. } \\
\text { E4+ had a larger difference in } \\
\text { amplitude between congruous and } \\
\text { incongruous odor-image pairs at right } \\
\text { ventral electrode sites than E4-, } t \\
\quad(9)=3.97, p=0.003 \text {. } \\
\text { ApoE E4 carriers had a significantly } \\
\text { smaller ERP amplitude difference than } \\
\text { non-carriers }(F(1,17)=5.12, p=0.037) \text {. }\end{array}$ & $\begin{array}{l}\text { A scalp topography of ApoE4 } \\
\text { carriers was consistent } \\
\text { with morphological and } \\
\text { hypometabolic abnormalities } \\
\text { found in PET, fMRI and MRI } \\
\text { studies. OERPs reflected } \\
\text { hemispheric asymmetries in } \\
\text { E4 carriers that were line with } \\
\text { a compensatory mechanism. } \\
\text { OERPs in an odor/visual } \\
\text { congruency task differentiated } \\
\text { ApoE4+ and ApoE4-. }\end{array}$ \\
\hline Corby et al. [97] & $\begin{array}{l}\text { Young and middle- } \\
\text { aged subjects with } \\
\text { E4 polymorphisms: } \\
\text { E4 + (20) } \\
\text { E4 - (20) }\end{array}$ & $\begin{array}{c}\text { E4 +: Young }=23.9+2.8 \text { Middle } \\
\text { age }=50.6+2.4 \\
E 4-: \\
\text { Young } 22.2+3.0 \text { Middle } \\
\text { age }=49.9+3.2\end{array}$ & $\begin{array}{c}\text { Chemosensory tests } \\
\text { with the butanol odor } \\
\text { threshold; } \\
\text { SDOIT; OERPs. }\end{array}$ & $\begin{array}{l}\text { Significant effects of ApoE status for } \mathrm{P} 3 \\
\text { latency collapsed across age }(\mathrm{F}(1,36)= \\
21.91, \mathrm{p}<0.001, \eta 2=0.38) \text {, with } \mathrm{ApoE} \\
\text { E4- participants demonstrating shorter } \\
\text { latencies than ApoE E4+ participants. } \\
\text { In the young group olfactory } \mathrm{P} 3 \\
\text { latency was the most significant } \\
\text { predictor }(\mathrm{X} 2=7.69, \mathrm{p} \text { b } 0.01 \text { ) resulting } \\
\text { in overall classification rate of } 75 \% \\
\text { (Sensitivity=80\%, Specificity }=70 \%) . \\
\text { In the middle age group olfactory } \mathrm{P} 3 \\
\text { latency was also the most significant } \\
\text { predictor }(\mathrm{X} 2=12.54, \mathrm{p}=0.001) \text { resulting } \\
\text { in overall classification rate of } 80 \% \\
\text { (Sensitivity=80\%, Specificity }=80 \%) .\end{array}$ & $\begin{array}{l}\text { OERP is sensitive to very } \\
\text { subtle changes in the brain } \\
\text { associated with the ApoE } \\
\text { E4 allele, even at much } \\
\text { younger ages than previously } \\
\text { demonstrated. Additionally, } \\
\text { the OERP is more sensitive to } \\
\text { these changes than traditional } \\
\text { tests of olfactory functioning. }\end{array}$ \\
\hline Handley et al. [90] & $\begin{array}{l}\text { Sibling group (24): } \\
E+(10) ; E-(14) \\
\text { Control group (47) } \\
E+(33) ; E-(14)\end{array}$ & $\begin{array}{l}\text { Sibling group mean age } \\
\text { (range): } 74.08(59-88) \\
\text { Control group: } 73.17(61-87)\end{array}$ & $\begin{array}{l}\text { Odor identification } \\
\text { performance test }\end{array}$ & $\begin{array}{l}\text { Lower odor identification scores for the } \\
\text { sibling group } 4.17 \pm 2.20 \text { compared } \\
\text { to control group } 5.60 \pm 2.22, F(1,67) \\
10.42, p<0.01 \text {. } \\
\text { No difference in odor identification } \\
\text { accuracy between } E 4+5.17 \pm 2.44 \text {, and } \\
\text { E4- groups } 5.09 \pm 2.44), p=n s \text {. } \\
\text { The group } \times \text { ApoE } E 4 \text { status interaction } \\
\text { was significant, } F(1,67) 6.10, p<0.05 \text {. } \\
\text { The sibling } E 4+\text { group had the poorest } \\
\text { mean odor identification scores, lower } \\
\text { than control } E 4+(t(22)=-4.12, p<0.01) \\
\text { and control } E 4-(t(44)=-2.33, p<0.05) \text {. }\end{array}$ & $\begin{array}{l}\text { Odor identification deficits } \\
\text { may reflect early disease } \\
\text { progression in individuals at } \\
\text { increased risk for developing } \\
\text { the disease. }\end{array}$ \\
\hline $\begin{array}{l}\text { Calhoun-Haney and } \\
\text { Murphy [83] }\end{array}$ & $\begin{array}{l}\text { Year } 1 \text { (baseline): } \\
\text { E4+ (22) } \\
\text { E- (28); } \\
\text { Year } 4 \text { (follow-up): } \\
\text { E4+ (22) } \\
\text { E- (28) }\end{array}$ & $\begin{array}{c}\text { Year } 1 \text { : } \\
\text { E4 + =73; } \\
\text { E4 - =71; } \\
\text { Year 4: } \\
\text { E4 + =77; } \\
\text { E4 - =75. }\end{array}$ & $\begin{array}{l}\text { Butanol odor } \\
\text { threshold test; } \\
\text { SDOIT }\end{array}$ & $\begin{array}{l}\text { At baseline there was no significant } \\
\text { difference between performance on } \\
\text { odor identification between the two } \\
\text { allele groups, }(F(1,48)=2.9, p=0.09) \text {, } \\
\text { although there was the trend toward } \\
\text { poorer performance in the } E 4+\text { group. } \\
\text { Allele status significantly affected } \\
\text { performance on the odor identification } \\
\text { measure at Year } 4 \text { follow-up, }(F(1, \\
48)=20.0, p<0.0005) \text {, with performance } \\
\text { significantly poorer in } E 4+\text { than in } E 4- \\
\text { individuals. E4+ individuals significantly } \\
\text { declined in odor identification } \\
\text { performance over time }(F(1,21)=22.9, \\
p<0.0005), \text { but } E 4-\text { individuals did not } \\
(F(1,27)=0.41, p=0.52) \text {. There was no } \\
\text { main effect of time }(F(1,48)=0.39, \\
p=0.54) \text { or allele status }(F(1,48)=1.7, \\
p=0.20) \text { on odor threshold. Detection of } \\
\text { odor at higher dilution steps indicates } \\
\text { better sensitivity. Neither the } E 4+\text { nor } \\
E 4-\text { group showed a significant decline } \\
\text { in global cognitive performance over } \\
\text { time. }\end{array}$ & $\begin{array}{l}\text { Study demonstrates that } \\
\text { normal non-demented elderly } \\
\text { adults who carry the E4+ } \\
\text { allele and are thus at risk } \\
\text { for AD, showed a significant } \\
\text { decline over a 4-year time } \\
\text { period in performance on } \\
\text { odor identification but not } \\
\text { on odor threshold, picture } \\
\text { identification or the DRS. } \\
\end{array}$ \\
\hline
\end{tabular}


Citation: Misiak MM, Hipolito MMS, Ressom HW, Obisesan TO, Manaye KF, et al. (2017) Apo E4 Alleles and Impaired Olfaction as Predictors of Alzheimer's Disease. Clin Exp Psychol 3: 169. doi: 10.4172/2471-2701.1000169

\begin{tabular}{|c|c|c|c|c|c|}
\hline $\begin{array}{l}\text { Wetter and Murphy } \\
\text { [94] }\end{array}$ & $\begin{array}{l}\mathrm{E} 4+(10) \\
\mathrm{E} 4-(10)\end{array}$ & $\begin{array}{l}E 4+=75.7 \pm 7.7 \\
E 4-=75.3 \pm 6.4\end{array}$ & $\begin{array}{l}\text { Amyl acetate odor } \\
\text { threshold test; } \\
\text { UPSIT; SDOIT; } \\
\text { OERPs. }\end{array}$ & $\begin{array}{c}\text { Significant delays for } E 4+\text { individuals at } \\
\text { each OERP component at: } \\
\text { N1 }[F(1,18)=17.8, p=0.001)] \text {, } \\
\text { P2 }[F(1,18)=19.7, p=0.001] \text {, } \\
\text { N2 }[F(1,18)=22.4, p=0.001] \text {, and } \\
\text { P3 }[F(1,18)=16.1, p=0.001 . \\
\text { Individuals with poorer ability to } \\
\text { identify odors also showed increased } \\
\text { olfactory P3 latencies, suggesting } \\
\text { that the ability to identify odors is } \\
\text { specifically associated with the speed } \\
\text { of cognitive processing of olfactory } \\
\text { stimuli. Psychophysical (UPSIT, and } \\
\text { odor threshold) and cognitive (DRS } \\
\text { and MMSE) measure revealed no } \\
\text { significant effects of allele status in this } \\
\text { sample size. }\end{array}$ & $\begin{array}{l}\text { ApoE4+ Individuals } \\
\text { demonstrated delays in } \\
\text { the processing of olfactory } \\
\text { information compared to } \\
\text { those who are E4-. OERP } \\
\text { latency appears to be more } \\
\text { sensitive for detecting } \\
\text { olfactory deficits than the } \\
\text { psychophysical measures } \\
\text { utilized in this study. }\end{array}$ \\
\hline Bacon et al. [86] & $E+(9) ; E-(6)$ & $\begin{array}{l}E+=75.75 \pm 5.01 \\
E-=80.67 \pm 5.39\end{array}$ & $\begin{array}{l}\text { Butanol odor } \\
\text { threshold; } \\
\text { MMSE; DRS }\end{array}$ & $\begin{array}{c}\text { E4+ performed worse on DRS: } t(12)= \\
-2.18, p=0.05 \text { and odor threshold. } \\
\text { E4+ participants showed poorer odor } \\
\text { sensitivity to butanol than E4-. } \\
\text { No differences between groups on the } \\
\text { MMSE }\end{array}$ & $\begin{array}{c}\text { First study documenting } \\
\text { differences in performance } \\
\text { on a test of olfaction in group } \\
\text { of adults at risk for AD (E4+), } \\
\text { more sensitive than MMSE. }\end{array}$ \\
\hline $\begin{array}{l}\text { Sundermann et al. } \\
{[87]}\end{array}$ & $\begin{array}{c}\mathrm{MCl} \text { patients } \\
\text { (converters and } \\
\text { nonconverters } \\
\text { to AD during 1-9 } \\
\text { years follow up): } \\
\text { Converters (39); } \\
\text { Nonconverters } \\
(109) ; \\
\text { Controls }(63)\end{array}$ & $\begin{array}{c}\text { converters }=73.2 \pm 7.1 \\
\text { Nonconverters }=64.9 \pm 9.9 \\
\text { Controls }=65.7 \pm 9.3\end{array}$ & $\begin{array}{l}\text { UPSIT; } \\
\text { Apolipoprotein E } \\
\text { genotype: patients } \\
\text { classified as E4+ } \\
\text { or E4-. }\end{array}$ & $\begin{array}{c}\text { UPSIT scores in: } \\
\text { Converters }=25.8 \pm 8.4 \\
\text { Non-converters }=33.2 \pm 4.6 \\
\text { Controls }=34.8 \pm 4.2 \\
\\
\% \text { of E4 carriers: converters }=34.3 \text {; non- } \\
\text { converters }=23.6 \text {; controls } 22.4\end{array}$ & $\begin{array}{l}\text { Clinical sample of cognitively } \\
\text { impaired, non-demented } \\
\text { patients exhibiting memory } \\
\text { complaints shows that ApoE } \\
\text { and olfactory dysfunction can } \\
\text { be combined for prediction of } \\
\text { conversion rate to } A D\end{array}$ \\
\hline $\begin{array}{l}\text { Gilbert and Murphy } \\
\text { [84] }\end{array}$ & $\begin{array}{l}\text { Control group (38): } \\
\text { E4+ (19); E4- (19) } \\
\text { Probable AD (38): } \\
\text { E4+ (24); E4- (14) } \\
\\
\text { Confirmed AD (38): } \\
\text { E4+ (23); E4- (15) }\end{array}$ & $\begin{array}{c}\text { Control: } \\
\text { E4+ =71.3 0 } \pm 2.24 ; \text { E4- }=71.44 \\
\pm 2.07 ; \text { Probable AD: } \\
\text { E4+ =74.15 } \pm 1.45 ; \text { E4- }=73.70 \\
\pm 1.93 ; \text { Confirmed AD: } \\
\begin{aligned} \mathrm{E} 4+=72.16 \pm 1.31 ; \mathrm{E} 4-=75.90 \\
\pm 2.40\end{aligned}\end{array}$ & $\begin{array}{l}\text { Odor threshold test; } \\
\text { Recognition memory } \\
\text { task developed by } \\
\text { Murphy. }\end{array}$ & $\begin{array}{c}\text { Significant effect of threshold for } \\
\text { group } F(2,108)=6.13, p<0.01 \text {, but no } \\
\text { significant effect for } E 4 \text { status or an } \\
\text { E4 status } x \text { group interaction, } p>0.05 \text {. } \\
\text { On the recognition memory task, E4- } \\
\text { controls committed significantly fewer } \\
\text { false positive errors than E4+ controls } \\
\text { or } A D \text { patients. E4+ controls did not } \\
\text { differ from } A D \text { patients in false positive } \\
\text { errors. }\end{array}$ & $\begin{array}{l}\text { The analysis of false positive } \\
\text { errors in recognition memory } \\
\text { for olfactory stimuli may be } \\
\text { useful in identifying early } \\
\text { deficits in cognition. }\end{array}$ \\
\hline $\begin{array}{l}\text { Gilbert and Murphy } \\
\text { [84] }\end{array}$ & $\begin{array}{c}\text { E4+ (21); } \\
\text { E4- (21) }\end{array}$ & $\begin{array}{c}E 4+=71.38 \pm 1.88 ; E 4-=71.45 \\
\pm 2.06\end{array}$ & $\begin{array}{l}\text { Odor threshold test; } \\
\text { Recognition Memory } \\
\text { Task by Murphy. }\end{array}$ & $\begin{array}{c}\text { No significant difference between the } \\
\text { odor thresholds of the } E 4+\text { and } E 4- \\
\text { individuals }(F(1,40)=0: 01 ; p=0.97) \text {. } \\
\text { No differences between the mean } \\
\text { number of hits committed by } E 4+ \\
\text { and } E 4 \text { - individuals on recognition } \\
\text { memory tasks involving odors, faces, } \\
\text { or symbols. } \\
\text { E4+ individuals committed more false } \\
\text { positive errors relative to } E 4 \text { - individuals } \\
\text { for olfactory stimuli, but not for faces or } \\
\text { symbols }\left(F(1,40)=4,22 ; p=0: 05^{\star}\right) \\
\text { No significant differences between } \\
E 4+\text { and } E 4-\text { individuals on } \\
\text { recognition memory tasks involving } \\
\text { odors } F(1,40)=0.01 ; p=0.44 ; \text { faces } \\
F(1,40)=2.20 ; p=0.15 ; \text { or symbols } F(1, \\
40)=1.98 ; p=0.28\end{array}$ & $\begin{array}{l}\text { The remote memory for } \\
\text { olfactory and visual stimuli } \\
\text { was not impaired in non- } \\
\text { demented E4+ individuals } \\
\text { compared to E4 positive } \\
\text { controls. These data suggest } \\
\text { that the areas of the brain } \\
\text { involved in retrieval of remote } \\
\text { memories are not significantly } \\
\text { affected in non-demented } \\
\text { individuals genetically at risk } \\
\text { for AD. }\end{array}$ \\
\hline Kjelvik et al. [89] & $\begin{array}{l}\text { AD and Controls } \\
\text { (\% carriers of E4 } \\
\text { alleles): } \\
\\
\text { Patients } n=18 ; E 4+ \\
(73.3 \%) \\
\\
\text { Controls } n=30 ; E 4+ \\
(20.8 \%)\end{array}$ & $\begin{array}{l}\text { Patients }=74.6 \pm 6.3 \\
\text { Controls }=67.4 \pm 7.6\end{array}$ & B-SIT; SSIT; SSDT & $\begin{array}{c}\text { Patients performed significantly worse } \\
\text { than healthy controls on the two odor } \\
\text { identification tests (B-SIT and SSIT), } \\
\text { but not on the odor discrimination test } \\
\text { SSDT. } \\
\text { B-SIT: controls - } 9.6 \pm 2.0 \text {, patients }-6.6 \\
\pm 2.6^{* \star} p<0.005 ; \text { SSIT: controls - } 12.7 \pm \\
2.4 \text {, patients - } 9.4 \pm 3.0^{* *} p<0.005 ; \\
\text { The B-SIT score did not differentiate } \\
\text { patients with aMCI from those with AD } \\
\text { at baseline ( } p>0.4 \text { ), but differentiated } \\
\text { those patients persisting with aMCI } \\
\text { from those who had progressed to AD } \\
6-18 \text { months later (mean } 9.9 \text { months, } \\
\text { t-test; } p=0.037 \text { ). SSIT scores did not } \\
\text { differentiate patients converting to AD. }\end{array}$ & $\begin{array}{l}\text { Competence in olfactory } \\
\text { identification was also } \\
\text { associated with the volume } \\
\text { of several brain structures, } \\
\text { particularly hippocampus, } \\
\text { more than scores on memory } \\
\text { tasks in aMCl and AD. } \\
\text { Olfactory tests distinguished } \\
\text { patients with aMCl and early } \\
\text { AD from healthy control } \\
\text { individuals, and suggested } \\
\text { that patients with greater } \\
\text { olfactory impairment have } \\
\text { increased brain atrophy. }\end{array}$ \\
\hline
\end{tabular}


Citation: Misiak MM, Hipolito MMS, Ressom HW, Obisesan TO, Manaye KF, et al. (2017) Apo E4 Alleles and Impaired Olfaction as Predictors of Alzheimer's Disease. Clin Exp Psychol 3: 169. doi: 10.4172/2471-2701.1000169

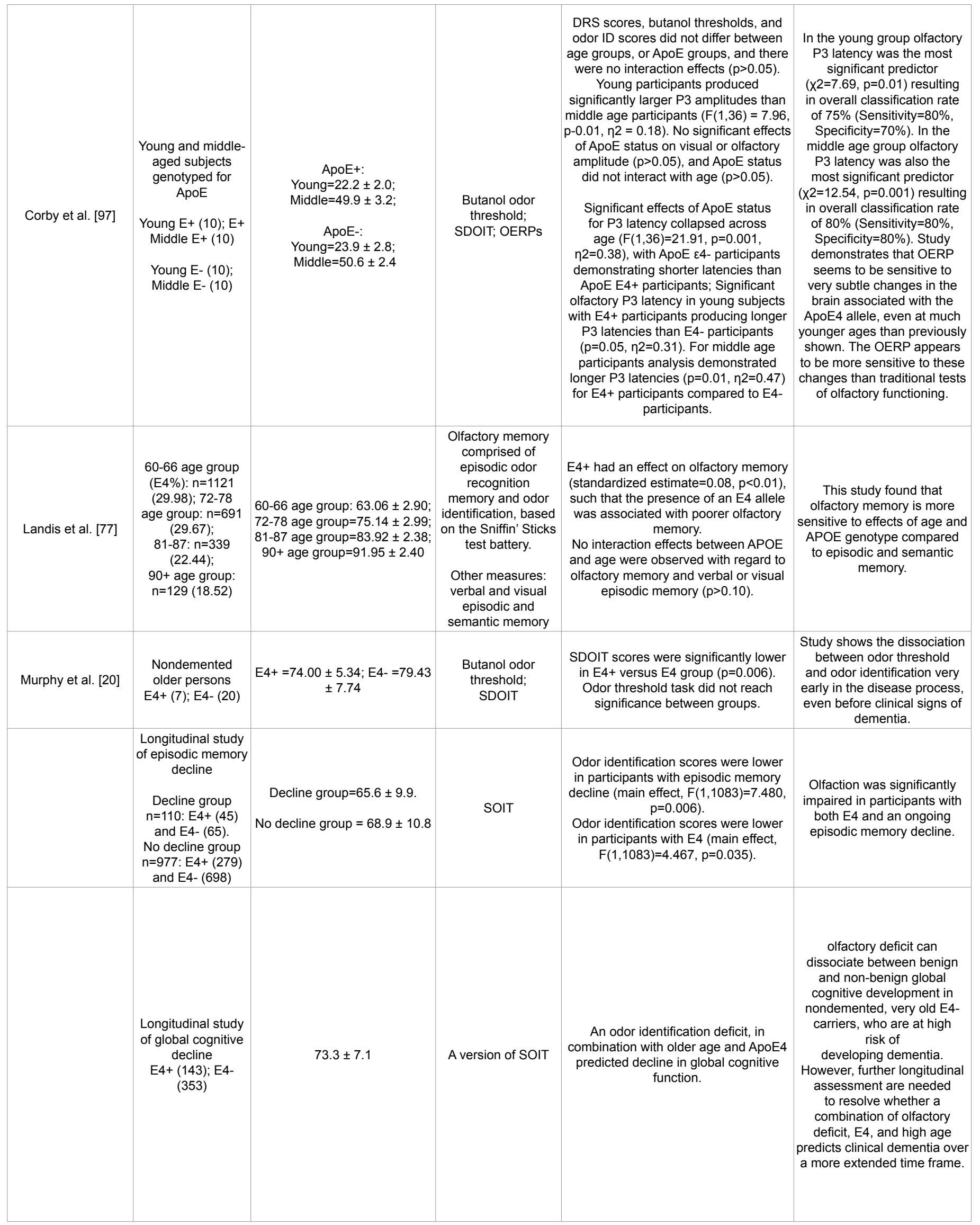


Citation: Misiak MM, Hipolito MMS, Ressom HW, Obisesan TO, Manaye KF, et al. (2017) Apo E4 Alleles and Impaired Olfaction as Predictors of Alzheimer's Disease. Clin Exp Psychol 3: 169. doi: 10.4172/2471-2701.1000169

\begin{tabular}{|c|c|c|c|c|c|}
\hline & $\begin{array}{l}\text { Population based } \\
\text { longitudinal study } \\
\begin{array}{c}\text { E4+ (372); E4- } \\
\text { (864) }\end{array}\end{array}$ & $\begin{aligned} E 4+=61.5 & \pm 11.3 ; E 4-=60.7 \\
& \pm 10.8\end{aligned}$ & A version SOIT & $\begin{array}{c}\text { Odor identification performance } \\
\text { decreased most strongly in older } \\
\text { participants with ApoE4; ApoE } 4 \times \text { age } \\
\text { interaction was significant }(p=0.033) \text {. } \\
\text { Although several demographic, } \\
\text { cognitive, and health variables affected } \\
\text { odor identification, this results suggest } \\
\text { that there was a unique effect of the } \\
\text { ApoE4 } \times \text { age interaction on odor } \\
\text { identification. Predictor variables } \\
\text { accounted for } 21.3 \% \text { of the explanatory } \\
\text { variance in odor identification } \\
\text { performance. }\end{array}$ & $\begin{array}{l}\text { The effect of ApoE4 on odor } \\
\text { identification in the } 75-80 \\
\text { age range is not driven by } \\
\text { individuals that receive a } \\
\text { dementia diagnosis within a } \\
5 \text {-year period after olfactory } \\
\text { assessment. The present } \\
\text { results suggest that the ApoE } \\
\text { gene plays a significant } \\
\text { role for the integrity of the } \\
\text { olfactory system in non- } \\
\text { demented, elderly individuals. }\end{array}$ \\
\hline & $\begin{array}{l}\text { Young E4+ (11); } \\
\text { Young E4- (13). } \\
\text { Old E4+ (14) } \\
\text { Old E4- (13) }\end{array}$ & $\begin{array}{l}\text { Young E4+ =22.1 } \pm 1.8 \\
\text { Young E4- }=23.1 \pm 2.7 \\
\text { Old E4+ =70.8 } \\
\text { Old E4- }=71.6 \pm 4.0\end{array}$ & $\begin{array}{l}\text { Odor threshold, } \\
\text { olfactory } \\
\text { ERP stimulus } \\
\text { presentation, } \\
\text { recording and } \\
\text { analysis }\end{array}$ & 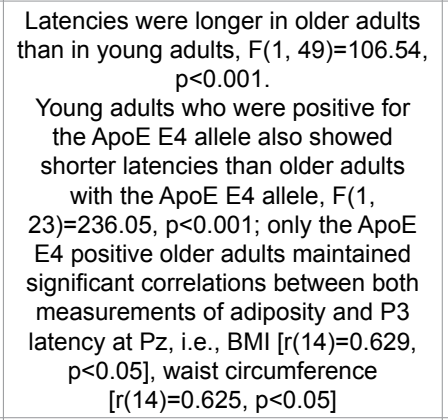 & $\begin{array}{l}\text { Study shows a positive linear } \\
\text { relationship between adiposity } \\
\text { and prolonged olfactory } \\
\text { latencies in older adults. } \\
\text { When analyzed separately, } \\
\text { this relationship remained } \\
\text { significant only in older adults } \\
\text { who were positive for the } \\
\text { ApoE4 allele. There were } \\
\text { no significant differences in } \\
\text { amplitude between groups. }\end{array}$ \\
\hline & E4+ (15); E4- (23) & $\begin{array}{c}E 4+=58.0 \pm 6.3 ; E 4-=58.0 \\
\pm 11.1\end{array}$ & B-SIT test & $\begin{array}{l}\text { B-SIT score }(\mathrm{M} \pm \mathrm{SD}) \text { in } E 4+v \text { vs. E4- } \\
=8.9 \pm 1.9 \text { and } 10.1 \pm 1.2, p<0.05\end{array}$ & $\begin{array}{c}\text { Higher occurrence of olfactory } \\
\text { dysfunction among Irish } \\
\text { individuals at genetic risk of } \\
\text { dementia. }\end{array}$ \\
\hline Sliger et al. [93] & $\begin{array}{l}\text { Down Syndrome } \\
\text { (DS) (34): E4+ } \\
\text { (12); E4- (22); } \\
\text { Control n } 34\end{array}$ & $\begin{array}{c}\mathrm{DS}=31.2 \pm 1.59 \\
\text { Control=31.3 } \pm 1.60\end{array}$ & SDOIT & $\begin{array}{l}\text { Participants with DS possessing } \\
\text { at least one E4 allele performed } \\
\text { significantly poorer on the odor } \\
\text { identification test compared to those } \\
\text { without the E4 allele. The mean number } \\
\text { of odors identified in the E4+ group was } \\
4.4 \text {, compared to } 5.7 \text { in the E4- group, } \\
\qquad F(1,33)=4.51, p=0.04 \text {. }\end{array}$ & $\begin{array}{l}\text { Individuals with DS carrying } \\
\text { the ApoE4 allele, exhibit } \\
\text { significantly greater deficits } \\
\text { in odor identification than } \\
\text { those who are negative for } \\
\text { the allele. }\end{array}$ \\
\hline $\begin{array}{l}\text { Morgan and Murphy } \\
\text { [99] }\end{array}$ & $\begin{array}{l}\text { Young E4+ (10); } \\
\text { Middle age E+ (10); } \\
\text { Old E4+ (10). } \\
\\
\text { Young E4- (10); } \\
\text { Middle age E4- } \\
\text { (10); Old E4- (10). }\end{array}$ & $\begin{array}{l}\text { Young E4+ =23.1 } \pm 2.3 \\
\text { Middle age E4+ }=50.2 \pm 4.5 \\
\text { Old E4+ } \\
\text { Young E4- }=22.6 \pm 2.0) \\
\text { Middle age E4- }=50.7 \pm 1.7 \\
\text { Old E4- }=71.2 \pm 3.6\end{array}$ & $\begin{array}{l}\text { Odor Threshold test; } \\
\text { SDOIT; OERPs }\end{array}$ & $\begin{array}{l}\text { SDOIT test revealed no significant main } \\
\text { effects or interaction effects involving } \\
\text { ApoE status }(p>0.05) \text {. Odor } \\
\text { ERP task revealed a main effect } \\
\text { of ApoE status collapsed across } \\
\text { age groups }(F(1,54)=4.54, p<0.05) \\
\eta 2=0.08) \text {. } \\
\text { Varying patterns of brain activation } \\
\text { were observed over the post-stimulus } \\
\text { epoch for E4- versus E4+ individuals } \\
\text { on topographical maps. Individuals with } \\
\text { the E4 allele demonstrated different } \\
\text { ERP peak latencies during identification } \\
\text { of olfactory but not visual stimuli. }\end{array}$ & $\begin{array}{l}\text { Olfactory ERPs detected } \\
\text { functional decline in } \\
\text { individuals at risk for } \\
\text { Alzheimer's disease at much } \\
\text { earlier ages than previously } \\
\text { observed, suggesting the } \\
\text { potential role of ERPs for pre- } \\
\text { clinical detection of AD at very } \\
\text { early stages. }\end{array}$ \\
\hline $\begin{array}{l}\text { Sundermann et al. } \\
{[87]}\end{array}$ & $\begin{array}{r}\text { Non-demented } \\
\text { Older Females: } \\
\text { E4+ on Hormonal } \\
\text { Therapy, HT }(n=8) ; \\
\text { E4+ No HT }(n=11) ; \\
\text { E4- on HT }(n=8) ; \\
\text { E4- No HT }(n=24) ; \\
\text { AD Female Group: } \\
\text { E4+ on HT }(n=12) ; \\
\text { E4+ No HT }(n= \\
\text { 35); E4- on HT } \\
(n=6 ; \\
\text { E4- No HT }(n=24) .\end{array}$ & $\begin{array}{c}\text { Non-demented Older Females: } \\
\text { E4+ on HT }=70.41 \pm 4.22 ; \\
\text { E4+ No HT }=69.70 \pm 3.60 ; \\
E 4-\text { on HT }=73.18 \pm 4.22 ; \\
\text { E4- No HT }=73.77 \pm 2.43 ; \\
\text { AD Females: } \\
\text { E4+ on HT =72.94 } \pm 2.61 ; \\
E 4+\text { No HT } 76.48 \pm 1.53 ; \\
\text { E4- on HT }=72.97 \pm 3.69 \\
\text { E4- No HT } 75.61 \pm 1.89\end{array}$ & $\begin{array}{l}\text { Butanol odor } \\
\text { threshold }\end{array}$ & $\begin{array}{l}\text { HT had no effect on olfactory sensitivity } \\
\text { in female AD patients regardless of E4 } \\
\text { genotype. Within the non-demented } \\
\text { no HT group, E4- females had a } \\
\text { significantly better threshold score than } \\
\text { E4+ females. No significant differences } \\
\text { existed in odor threshold scores } \\
\text { between the E4+ and E4- females in } \\
\text { the HT comparison group. }\end{array}$ & $\begin{array}{l}\text { HT may exert neuroprotective } \\
\text { effects on brain areas affected } \\
\text { by } A D . H T \text { is protective } \\
\text { against loss of odor sensitivity } \\
\text { function in E4 positive } \\
\text { individuals in preclinical } \\
\text { stages of AD. }\end{array}$ \\
\hline
\end{tabular}


Citation: Misiak MM, Hipolito MMS, Ressom HW, Obisesan TO, Manaye KF, et al. (2017) Apo E4 Alleles and Impaired Olfaction as Predictors of Alzheimer's Disease. Clin Exp Psychol 3: 169. doi: 10.4172/2471-2701.1000169

\begin{tabular}{|c|c|c|c|c|c|}
\hline Murphy et al. [82] & $\begin{array}{l}20 \text { Non-demented } \\
\text { Old Adults: } \\
\text { E4+ (10); E4- (10) }\end{array}$ & $\begin{array}{c}E 4+=75.1 \pm 8.3 \\
E 4-=71 \pm 6.1\end{array}$ & $\begin{array}{l}\text { Odor Threshold Test; } \\
\text { SDOIT; OERP; EEG }\end{array}$ & $\begin{array}{c}\text { No significant difference in odor } \\
\text { threshold scores between ApoE4+ and } \\
\text { E4- participants. } \\
\text { P3 latency was significantly delayed } \\
\text { in ApoE E4+ individuals, } F(1,18)=9.6, \\
\text { p<0.05). The results support significant } \\
\text { differences between E4+ and E4- } \\
\text { individuals in P3 latency and in intra- } \\
\text { class correlations of activity. ApoE E4+ } \\
\text { individuals demonstrated significantly } \\
\text { longer P3 latency than E4+ individuals } \\
\text { and differential activity for all response } \\
\text { types. Differential activity in ApoE } \\
\text { E4+and E4- individuals, demonstrated } \\
\text { by the intra-class correlation } \\
\text { coefficients, is consistent with a } \\
\text { compensatory hypothesis, which posits } \\
\text { that E4+ individuals expend greater } \\
\text { effort in cognitive processing and } \\
\text { therefore require greater activation of } \\
\text { neural tissue during retrieval attempts. }\end{array}$ & $\begin{array}{l}\text { Study suggest that cross- } \\
\text { modal ERP studies of } \\
\text { recognition memory in } \\
\text { ApoE4+and E4- individuals } \\
\text { are a useful measure for } \\
\text { indexing functional brain } \\
\text { integrity, for understanding } \\
\text { the neurocognitive changes } \\
\text { associated with the ApoE4 } \\
\text { allele, and for discriminating } \\
\text { between brain response in } \\
\text { E4+ and E4- individuals. }\end{array}$ \\
\hline $\begin{array}{c}\text { Oleson and Murphy } \\
\text { [91] }\end{array}$ & $\begin{array}{l}\text { Older adults with } \\
\text { Probable AD } \\
\text { Experiment } 1 \\
(n=51): E 3 / 3 \\
(n=17) ; E 3 / 4 \\
(n=17) ; E 4 / 4 \\
(n=17) .\end{array}$ & $\begin{array}{c}\text { Experiment } 1: \\
\text { E3/3=74.38 } \pm 6.91 ; \\
\text { E3/4=74.71 } \pm 6.84 ; \text { E4/4=74.88 } \\
\pm 6.49 \\
\\
\text { Experiment } 2 \text { and } 3: \\
\text { E3/3=73.78 } \pm 7.08 ; \\
\text { E3/4=73.17 } \\
\pm 6.89 ; E 4 / 4=73.52 \\
\pm 6.59\end{array}$ & \begin{tabular}{|l} 
\\
\\
\\
Exp. 1: SDOIT \\
Exp. 2: Remote Odor \\
Memory Task \\
Exp. 2, 3: Odor \\
Threshold. \\
\end{tabular} & 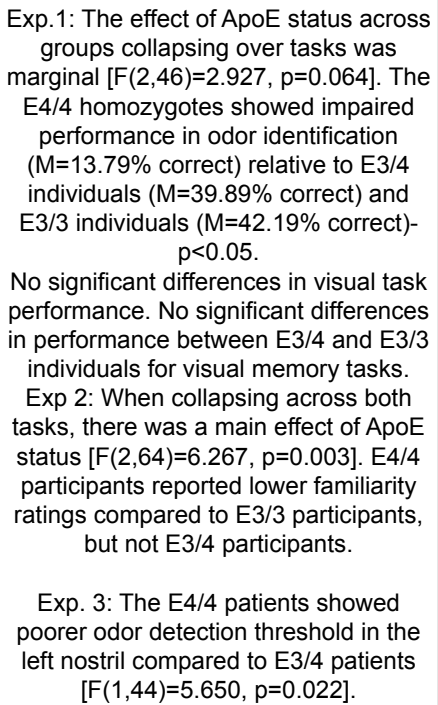 & $\begin{array}{l}\text { Percent correct odor } \\
\text { identification scores for AD } \\
\text { patients were noticeably } \\
\text { lower than scores for the E3/4 } \\
\text { group in the current study, } \\
\text { suggesting that combining } \\
\text { individuals with different } \\
\text { levels of } E 4 \text { allele status in a } \\
\text { sample of } A D \text { individuals may } \\
\text { show muted effects of } A D \text { on } \\
\text { olfactory function. }\end{array}$ \\
\hline
\end{tabular}

Note: AD Alzheimer's disease, aMCI amnestic mild cognitive impairment, APOE apolipoprotein E, BOLD blood-oxygen-level dependent, B-SIT brief smell identification test, CC-SIT cross-cultural smell identification test, CDR clinical dementia rating, DRS2 dementia rating scale 2, DS Down syndrome, E4 + APOE with presence of at least one E4 allele, E4 - APOE without any E4 allele, EEG electroencephalography, fMRI functional magnetic resonance imaging, HC healthy control, HT hormone therapy, MMSE mini-mental status examination, MRI magnetic resonance imaging, OERPs olfactory and visual event-related potentials, POC primary olfactory cortex, PET positron emission tomography, SDOIT San Diego odor identification test, SOIT Scandinavian odor identification test, SSDT Sniffin sticks discrimination test, SSIT Sniffin sticks identification test, UPSIT University of Pennsylvania smell identification test

Table 1: ApoE olfaction studies.

\begin{tabular}{|c|c|c|c|c|c|}
\hline $\begin{array}{c}\text { Author } \\
\text { (year) }\end{array}$ & Animal model & Age (months) & Measure & Findings & Conclusion \\
\hline $\begin{array}{c}\text { Nathan et al. } \\
\text { [101] }\end{array}$ & $\begin{array}{l}\text { ApoE KO mice; } \\
\text { WT C57BL/6 } \\
\text { strain }\end{array}$ & 4 months & BFP test; OC test; OCTA test. & $\begin{array}{l}\text { ApoE KO mice performed poorly in all three } \\
\text { tests compared to WT mice, while they } \\
\text { learned the tasks at a rate comparable to WT } \\
\text { mice. Latency to find the buried pellet was } \\
\text { significantly longer in ApoE KO mice than WT } \\
\text { mice. ApoE KO mice did not differentiate the } \\
\text { odorant and failed the avoidance test. }\end{array}$ & $\begin{array}{l}\text { ApoE deficiency in ApoE } \\
\text { KO mice leads to a deficit in } \\
\text { olfactory function, suggesting } \\
\text { an important role for ApoE in } \\
\text { the olfactory system. }\end{array}$ \\
\hline $\begin{array}{c}\text { Nathan et al. } \\
\text { [103] }\end{array}$ & $\begin{array}{l}\text { ApoE KO mice; } \\
\text { WT C57BL/6 } \\
\text { strain }\end{array}$ & 2-3 months & $\begin{array}{l}\text { OE Lesion; IHC of OB tissue }(0,3,7 \\
21,42, \text { and } 56 \text { days post-lesion) }\end{array}$ & $\begin{array}{c}\text { Slow OMP recovery in the OB in ApoE } \\
\text { KO compared to WT mice. Recovery of } \\
\text { glomerular area was similarly slower. GAP43 } \\
\text { accumulation and restore in the OB were } \\
\text { slower in KO mice. }\end{array}$ & $\begin{array}{l}\text { Olfactory nerve regeneration is } \\
\text { significantly slower in KO mice, } \\
\text { suggesting ApoE participates in } \\
\text { olfactory nerve regeneration. }\end{array}$ \\
\hline $\begin{array}{l}\text { MC Asey et } \\
\text { al. [113] }\end{array}$ & $\begin{array}{l}\text { ApoE KO mice; } \\
\text { WT C57BL/6 } \\
\text { strain }\end{array}$ & 2-4 months & $\begin{array}{l}\text { Ovariectomy. } \\
\text { Estradiol replacement. IHC of } \\
\text { Olfactory tissues }(5,14,28 \text { and } \\
49 \text { days after OVX and pellet } \\
\text { replacement). }\end{array}$ & $\begin{array}{l}\text { GFAP concentrations were higher in the } \\
\text { E2-deprived mice but did not increase in the } \\
\text { E2-replaced group at } 49 \text { days. Syn and ApoE } \\
\text { concentrations were significantly } \uparrow \text { by } 15 \% \\
\text { and } 25 \% \text {, respectively, in the E2-replaced } \\
\text { compared to the vehicle-replaced group at } \\
5 \text { days, but by } 14 \text { days concentrations were } \\
\text { equivalent. }\end{array}$ & $\begin{array}{l}\text { Estradiol is able to suppress } \\
\text { reactive gliosis. In addition, } \\
\text { E2 replacement in OVX mice } \\
\text { is associated with transiently } \\
\text { higher levels of ApoE and Syn. }\end{array}$ \\
\hline
\end{tabular}


Citation: Misiak MM, Hipolito MMS, Ressom HW, Obisesan TO, Manaye KF, et al. (2017) Apo E4 Alleles and Impaired Olfaction as Predictors of Alzheimer's Disease. Clin Exp Psychol 3: 169. doi: 10.4172/2471-2701.1000169

\begin{tabular}{|c|c|c|c|c|c|}
\hline $\begin{array}{c}\text { Nathan et al. } \\
\text { [102] }\end{array}$ & $\begin{array}{l}\text { ApoE KO mice; } \\
\text { WT C57BL/6 } \\
\text { strain }\end{array}$ & 4 months & $\begin{array}{l}\text { IHC staining of OB and OE tissues } \\
\text { fpr ApoE iimunoreactivity }\end{array}$ & $\begin{array}{c}\text { The perikarya and processes of sustentacular } \\
\text { (Sus) cells expressed ApoE-like } \\
\text { immunoreactivity. } \\
\text { The endothelial cells of blood vessels were } \\
\text { intensely stained for ApoE in the lamina } \\
\text { propria. Cells forming Bowman's gland also } \\
\text { immunostained for ApoE. The ApoE staining } \\
\text { in the nerve fascicles was less intense, but } \\
\text { was uniformly distributed throughout the } \\
\text { core of the nerve bundles. Ensheathing glia, } \\
\text { surrounding the nerve fascicles also stained } \\
\text { heavily for ApoE. }\end{array}$ & $\begin{array}{l}\text { ApoE is expressed in } \\
\text { the adult OE and lamina } \\
\text { propria at strategic locations } \\
\text { where it could facilitate the } \\
\text { differentiation, maturation and } \\
\text { axonal growth of the ORN, } \\
\text { perhaps by recycling lipids from } \\
\text { degenerating ORN for use by } \\
\text { growing axons. }\end{array}$ \\
\hline $\begin{array}{c}\text { Cheng et al. } \\
\text { [112] }\end{array}$ & $\begin{array}{l}\text { ApoE KO mice; } \\
\text { WT C57BL/6 } \\
\text { strain }\end{array}$ & 2-4 months & $\begin{array}{l}\text { Ovariectomy; } \\
\text { Estradiol (E2) replacement; IHC } \\
\text { staining of olfactory tissues. }\end{array}$ & $\begin{array}{c}\text { Five days of E2 replacement significantly } \uparrow \\
\text { LRP expression in the hippocampus, OB and } \\
\text { neocortex but not in cerebellum. } \\
\text { In contrast, E2 treatment } \downarrow \text { LRP expression } \\
\text { in OB. }\end{array}$ & $\begin{array}{l}\text { Hormone therapy }(\mathrm{HT}) \\
\text { modification of both ApoE and } \\
\text { LRP could have wide-spread } \\
\text { effects on cellular function } \\
\text { given LRP's manifold signaling } \\
\text { functions. }\end{array}$ \\
\hline $\begin{array}{c}\text { Nwosu et al. } \\
\text { [105] }\end{array}$ & $\begin{array}{l}\text { ApoE KO mice; } \\
\text { WT C57BL/6 } \\
\text { strain }\end{array}$ & $2-4$ months & $\begin{array}{l}\text { OE Lesion; } \\
\text { IHC and IB staining of OB tissue }(0, \\
3,7,21,42, \text { and } 56 \text { days post-nasal } \\
\text { irrigation). }\end{array}$ & $\begin{array}{c}\text { Sharp } \downarrow \text { in concentrations of Syn in OB } \\
\text { following injury in both WT and KO mice } \\
\text { during the degenerative phase ( } 3-7 \text { days). } \\
\text { Syn concentration in KO mice did not } \\
\text { recover by day } 56 \text { whereas Syn density in } \\
\text { WT was essentially restored to normal. IHC } \\
\text { of glomerular Syn density revealed a lower } \\
\text { density in KO mice at all-time points post } \\
\text { lesion. Lower concentration of whole bulb Syn } \\
\text { parallels the slower recovery of glomerular } \\
\text { area in KO mice. }\end{array}$ & $\begin{array}{c}\text { In the absence of ApoE, } \\
\text { synaptic recovery in whole } \\
\text { bulb samples is substantially } \\
\text { delayed compared to WT mice. }\end{array}$ \\
\hline $\begin{array}{c}\text { Nathan et al. } \\
\text { [106] }\end{array}$ & $\begin{array}{l}\text { ApoE KO mice; } \\
\text { WT C57BL/6 } \\
\text { strain }\end{array}$ & $2-4$ months & $\begin{array}{l}\text { OE Lesion; } \\
\text { Tissue preparation }(0,3,7,21,42, \\
\text { and } 56 \text { days post-treatment); IB; IHC }\end{array}$ & $\begin{array}{l}\text { ApoE expression in the OE is highly regulated } \\
\text { during the entire course of OE reconstitution } \\
\text { post injury, and ApoE deficiency in ApoE KO } \\
\text { mice leads to delayed recovery of mature } \\
\text { OMP+ cells in the reconstituting OE. }\end{array}$ & $\begin{array}{c}\text { ApoE production increases } \\
\text { in the injured OE to facilitate } \\
\text { maturation of olfactory sensory } \\
\text { neurons. }\end{array}$ \\
\hline $\begin{array}{l}\text { Nathan et al. } \\
\text { [106] }\end{array}$ & $\begin{array}{l}\text { ApoE KO mice; } \\
\text { WT C57BL/6 } \\
\text { strain }\end{array}$ & 4 months & $\begin{array}{l}\text { Ovariectomy; } \\
\text { BrdU injections, Olfactory turbinates } \\
\text { tissues; IHC }\end{array}$ & $\begin{array}{c}3 \text { days of estradiol replacement } \uparrow \text { ApoE } \\
\text { expression in the olfactory nerve and in the } \\
\text { glomerular layer. Estradiol treatment also } \uparrow \text { cell } \\
\text { proliferation, total cell numbers, number of } \\
\text { mature neurons in the olfactory epithelium, } \\
\text { and reactive astrocyte numbers in the OB in } \\
\text { both WT and KO mice. } \\
\text { Estradiol } \uparrow \text { glomerular synaptophysin (Syn), } \\
\text { but the magnitude of increase was potentiated } \\
\text { by the presence of ApoE. }\end{array}$ & $\begin{array}{l}\text { ApoE may be required to elicit } \\
\text { the complete effect of estradiol } \\
\text { on Syn upregulation. }\end{array}$ \\
\hline $\begin{array}{c}\text { Nathan et al. } \\
\text { [110] }\end{array}$ & $\begin{array}{l}\text { ApoE KO mice; } \\
\text { WT C57BL/6 } \\
\text { strain }\end{array}$ & 2-4 months & $\begin{array}{c}\text { Ovariectomy; } \\
\text { BrdU injections, Olfactory tissues; } \\
\text { IHC }\end{array}$ & $\begin{array}{c}\text { Estradiol replacement } \uparrow \text { ApoE staining in } \\
\text { the olfactory nerve and glomerular layers. } \\
\text { Estradiol } \uparrow \text { astrocyte density and OE thickness } \\
\text { regardless of the genotype. Estradiol } \\
\text { treatment } \uparrow \text { the number of mature neurons in } \\
\text { the OE and glomerular synaptophysin in both } \\
\text { genotypes, but the magnitude of increase was } \\
\text { greater in the WT than in the KO mice. }\end{array}$ & $\begin{array}{l}\text { Estrogen and ApoE act } \\
\text { synergistically to minimize the } \\
\text { loss of mature sensory neurons } \\
\text { and synapses following } \\
\text { ovariectomy. }\end{array}$ \\
\hline $\begin{array}{l}\text { Hussain et al. } \\
\text { [104] }\end{array}$ & $\begin{array}{l}\text { ApoE KO mice; } \\
\text { WT C } 57 \mathrm{BL} / 6 \mathrm{~J} \\
\text { mice }\end{array}$ & $\begin{array}{l}\text { post-natal pups } \\
\text { ( } 2 \text { days old })\end{array}$ & $\begin{array}{l}\text { Olfactory explant epithelial culture; } \\
\text { Immunocytochemistry; Measurement } \\
\text { of neuronal numbers, halo size, and } \\
\text { neurite outgrowth }\end{array}$ & $\begin{array}{l}\text { The OE cultures derived from ApoE KO mice } \\
\text { have significantly } \downarrow \text { neurons with shorter } \\
\text { neurite outgrowth than cultures from WT } \\
\text { mice. Treatment with either purified human } \\
\text { ApoE2 or with human ApoE3, but not } \\
\text { ApoE4, significantly } \uparrow \text { neurite outgrowth. The } \\
\text { differential effects of human ApoE isoforms on } \\
\text { neurite outgrowth were abolished by blocking } \\
\text { the LRP with lactoferrin and RAP. }\end{array}$ & $\begin{array}{l}\text { ApoE2 and ApoE3 stimulate } \\
\text { neurite outgrowth in } \mathrm{OE} \\
\text { cultures by interacting } \\
\text { with the LRP. ApoE4, the } \\
\text { isoform associated with } A D \text {, } \\
\text { failed to promote neurite } \\
\text { outgrowth, suggesting a } \\
\text { potential mechanism whereby } \\
\text { apoE4 may lead to olfactory } \\
\text { dysfunction in AD patients. }\end{array}$ \\
\hline $\begin{array}{l}\text { Peng et al. } \\
\text { [100] }\end{array}$ & $\begin{array}{l}\text { knock-in mice } \\
\text { humanized to } \\
\text { ApoE4 versus } \\
\text { ApoE3 }\end{array}$ & $\begin{array}{l}6 \text { months; } \\
12 \text { months }\end{array}$ & $\begin{array}{l}\text { Olfactory Perceptual Memory; in vivo } \\
\text { resting and odor-evoked local field } \\
\text { potentials (LPF) }\end{array}$ & $\begin{array}{c}\text { Young ApoE4 compared to ApoE3 mice } \\
\text { exhibited a behavioral olfactory deficit } \\
\text { coinciding with hyperactive odor-evoked } \\
\text { response magnitudes within the OB that were } \\
\text { not observed in older ApoE4 mice; shift with } \\
\text { aging in ApoE4-driven effects from OB to } \\
\text { PCX; }\end{array}$ & $\begin{array}{l}\text { Early ApoE4-driven olfactory } \\
\text { memory impairments and OB } \\
\text { network abnormalities may be } \\
\text { a precursor to later network } \\
\text { dysfunction in the PCX, a } \\
\text { region that not only is targeted } \\
\text { early in } A D \text {, but may be } \\
\text { selectively vulnerable to ApoE4 } \\
\text { genotype. }\end{array}$ \\
\hline
\end{tabular}

Note: AD Alzheimer's disease, BFP buried food pellet, BrdU bromodeoxyuridine, GAP 43 growth associated protein 43 , GFAP Glial fibrillary acidic protein, IB immunoblotting, IHC immunohistochemistry, LRP low-density lipoprotein (LDL) receptor related protein, OB olfactory bulb, OC odor choice, OCTA odor cued taste avoidance, OE olfactory epithelium, OMP olfactory marker protein, ORN olfactory receptor neuron, OVX ovariectomized, PCX piriform cortex, RAP receptor-associated protein, Syn synaptophysin (a synaptic marker).

Table 2: ApoE mice studies. 
[20,86]. Non-demented carriers of ApoE4 polymorphism showed significant decline in olfactory processing as compared to individuals without ApoE4 allele [87,88]. Importantly, patients with increased brain atrophy have greater olfactory impairment [89], indicating that olfactory function is further diminished as $\mathrm{AD}$ progresses. In family studies, siblings of $\mathrm{AD}$ probands had lower olfactory identification scores compared to siblings of control probands [90]. Moreover, within families, siblings with ApoE4 allele showed greater deficits in odor identification tests than siblings without ApoE4 allele. This finding of cosegregation between ApoE4 and olfactory identification deficits suggests that odor identification deficits may reflect early disease expression in individuals at increased risk for developing the disease [90]. Data from longitudinal studies provide additional supporting evidence of the association between ApoE4 inheritance and poor scores in olfactory tests, but more importantly highlights the predictive effect of baseline ApoE4 status on progression of olfactory loss over a 4-year period [83]. A recent study suggests that domains of high-order olfactory functioning, like odor identification and remote memory measured by odor familiarity ratings, may be more impaired in $\mathrm{AD}$ E4/4 homozygotes compared to E3/4 heterozygotes and E3/3 homozygotes [91]. These deficits give insight into how the presence of two E4 alleles may differentially affect the progression of AD. Down Syndrome involves trisomy of chromosome 21, where the gene for amyloid precursor protein (APP) is located. Down Syndrome (DS) represents premature aging disorder and autopsy studies show that by age 40 ; the brains of almost all individuals with DS have significant levels of plaques and tangles, which are definitive neuropathological features of $\mathrm{AD}$. These consistent brain changes notwithstanding, development of $\mathrm{AD}$ is not uniform in DS [92]. Interestingly, individuals with Down Syndrome (DS) who are carriers of ApoE4 allele, exhibit significantly greater deficits in odor identification than those who are negative for the allele [93]. It implies therefore, that olfactory tests and ApoE4 genotyping may contribute to improved prediction of AD risks in DS populations.

Olfactory event-related potentials (OERPs) have also demonstrated high sensitivity to subtle changes in olfactory functioning, and to $\mathrm{AD}$ and ApoE status [94-96]. A growing number of clinical studies have shown that brain changes associated with ApoE4 allele are captured much earlier in age through OERP recordings than through psychophysical tests of olfaction, thereby suggesting the potential utility of OERP for identification of preclinical stages of $\mathrm{AD}$ [82,97-99].

\section{Mechanistic studies linking ApoE4 to olfactory function}

Despite consistent evidence of the robust relationship between olfactory dysfunction, ApoE4 inheritance and $\mathrm{AD}$, the mechanism underlying these relationships are not fully understood. However, murine models of ApoE are beginning to illuminate our understanding of the role of ApoE in olfactory structure and function. The results of mechanistic studies in mice and from in vitro biochemical assays are highlighted in Table 2. ApoE deficiency in apoE $\mathrm{KO}$ mice leads to deficits in several tasks of olfactory function, suggesting an important role of ApoE in the mice olfactory system [100,101]. Previous studies showed that ApoE is expressed at high levels by a variety of cell types in the olfactory epithelium (OE). In particular, high expression of ApoE in the basal cells and adjoining lamina propria of the OE suggests that ApoE may play a role in the differentiation, maturation and axonal growth of ORNs, perhaps by recycling lipids from degenerating ORN for use by growing axons [102]. Indeed, studies of ApoE KO mice provide support that ApoE plays an important role in olfactory nerve regeneration [103]. Another group of investigators examined the effects of ApoE isoforms on neuronal differentiation and neurite outgrowth in OE explant cultures [104]. They discovered that ApoE2 and apoE3 stimulate neurite outgrowth in OE cultures by interacting with the lipoprotein receptor, LRP. ApoE4, the isoform associated with $\mathrm{AD}$, failed to promote neurite outgrowth, signifying a potential mechanism whereby ApoE4 may lead to olfactory dysfunction in $\mathrm{AD}$ patients [104]. Moreover, Nathan et al. [101] investigated the involvement of ApoE in propagating regeneration of $\mathrm{OE}$ cells by inducing OE lesions in ApoE and WT mice [101]. The results revealed that ApoE expression in the $\mathrm{OE}$ is highly regulated during the entire course of $\mathrm{OE}$ reconstitution post injury, and that ApoE deficiency in ApoE KO mice leads to delayed recovery of mature OMP+ cells in the reconstituting OE [105]. Glomerular Synaptophysin (Syn) density, measured by immunohistochemistry, was lower in $\mathrm{KO}$ mice at all time points following the lesion [105]. This lower concentration of whole bulb Syn paralleled the slower recovery of glomerular area in KO mice. In the absence of ApoE, synaptic recovery in whole bulb samples was significantly delayed compared to WT mice [106]. This study highlights the important role of ApoE in neuronal differentiation. It is noteworthy that ApoE has also been shown to modulate other molecular factors that are important for neurogenesis, including WNT2 and granulin [107-109]. Some studies have identified a close relationship between estrogen and apolipoprotein E (ApoE) in the central nervous system, and neuroprotective the role of hormone therapy (HT) in several neurological disorders. Estrogen and ApoE function synergistically to minimize the loss of mature sensory neurons and synapses in $\mathrm{OB}$, and OE following ovariectomy [110-113].

\section{Gaps in current studies and future directions}

Current diagnosis of Alzheimer's disease (AD) is based on clinical examination, neuropsychological testing and brain imaging; however a definite diagnosis can only be made by postmortem examination. Although brain imaging and cerebrospinal fluid biomarkers are applied in patients with mild or questionable symptoms to increase the level of diagnostic certainty, and peripheral bio-fluids are largely investigated, no definitive diagnostic tests are available yet. Biomarkers that reliably predict development of $\mathrm{AD}$ would greatly assist preventative and management treatments. This review demonstrates the potential relevance of olfactory system for both biomarker and pathophysiology studies of $\mathrm{AD}$ progression. However, many questions must still be answered. It is unclear how to identify and differentiate age-related olfactory changes and olfactory dysfunction caused by disease. Smell loss is also associated with other neurodegenerative disorders, like Parkinson's disease. Olfactory testing would need to be used with other biomarkers, specific to each disease, or olfactory changes in each disease have to be better specified. While the role of ApoE in olfactory neurogenesis has been reasonably demonstrated in basic studies, it remains uncertain how ApoE4 promotes $\mathrm{AD}$ amyloid and tau pathology in the olfactory system, especially in $\mathrm{OB}$ tissue. There are numerous studies examining interactions between ApoE4 protein and Amyloid or Tau proteins in cortical and hippocampal tissues [114]. ApoE is thought to be involved in plaque formation, but how exactly ApoE is involved in pathogenesis of $\mathrm{AD}$ is not well understood. The hypothesis gaining widespread support is that ApoE is involved in deposition or clearance of Abeta by direct protein-to-protein interaction. When associated with lipid, ApoE4 bound preferentially to an intermediate aggregated form of Abeta and had higher avidity than did ApoE2 or ApoE3 [115]. The tenability of this hypothesis in olfactory tissues has not been studied. Moreover, mechanistic correlations between ApoE and olfaction in $\mathrm{AD}$ to date are performed in animal models, not in humans. There are substantial differences between olfactory systems in rodents and 
Citation: Misiak MM, Hipolito MMS, Ressom HW, Obisesan TO, Manaye KF, et al. (2017) Apo E4 Alleles and Impaired Olfaction as Predictors of Alzheimer's Disease. Clin Exp Psychol 3: 169. doi: 10.4172/2471-2701.1000169

humans. Further research is necessary to clarify these uncertainties. Patient-derived olfactory neurons offer an excellent alternative tool to study correlations between ApoE4 and olfactory impairment. Moreover, these olfactory cells can be collected from people at high risk (e.g. ApoE4 carriers), particularly those who display progressive impairments in psychophysical and physiological tests of olfaction. With future accomplishment of these goals in mind, it is reasonable to anticipate that combination of psychophysical, neuroimaging, electrophysiological and molecular studies of olfactory tissues may hold promise for characterization preclinical stages of the disease in people at risk, such as the case of ApoE4 inheritance.

\section{Conclusion}

This review summarizes the research showing that ApoE4 is a significant player in olfactory impairment, an early $\mathrm{AD}$ symptom. Furthermore, we discussed the mechanistic studies that have been evaluated in ApoE KO mice. Our review stresses the importance of olfactory function as a biomarker of $\mathrm{AD}$, and a potential useful test for prediction of $\mathrm{AD}$ development in those with genetic (i.e., ApoE4) risk. Olfactory tests should be incorporated in the assessment of populations at high risk for dementia, like ApoE4 alleles for early recognition of $\mathrm{AD}$ and successful intervention.

\section{Disclaimer}

Sources of Funding/Disclaimers This project has been funded in part with Federal funds from the National Cancer Institute, National Institutes of Health, unde Contract No. HHSN261200800001E. Funding support for olfactory testing, and for the enrollment and neuropsychological testing of the control subjects is through USPHS grant $\mathrm{MH}-091460$ (PI, Nwulia). This publication does not necessarily reflect the views of policies of the Department of Health and Human Services nor does mention of trade names, commercial products, or organizations imply endorsement by the U.S. Government. Disclaimer: Dr. Kapetanovic contributed to this article as part of his official duties while working in the NIMH Intramural Office.

\section{Acknowledgement}

We are grateful to Ms. McLean and Dr. Rai at The Translational Neuroscience Laboratory of Howard University, for their assistance with the editorial changes made to this review article.

\section{References}

1. Corder EH, Saunder AM, Strittmatter WJ, Schmechel DE, Gaskell PC, et al. (1993) Gene dose of apolipoprotein E type 4 allele and the risk of Alzheimer's disease in late onset families. Science 261: 921-923.

2. Corder EH, Lannfelt L, Bogdanovic N, Fratiglioni L, Mori H (1998) The role of APOE polymorphisms in late-onset dementias. Cell Mol Life Sci 54: 928-934.

3. Ohm TG, Scharnagl H, Marz W, Bohl J (1999) Apolipoprotein E isoforms and the development of low and high Braak stages of Alzheimer's disease-related lesions. Acta Neuropathol 98: 273-280.

4. Farrer LA, Cupples LA, Haines JL, Hyman B, Kukull WA, et al. (1997) Effects of age, sex, and ethnicity on the association between apolipoprotein $E$ genotype and Alzheimer disease. A meta-analysis. APOE and Alzheimer Disease Meta Analysis Consortium. JAMA 278: 1349-1356

5. Bertram L, Tanzi RE (2005) The genetic epidemiology of neurodegenerative disease. J Clin Invest 115: 1449-1457.

6. Ueki A, Kawano M, Namba Y, Kawakami M, Ikeda K (1993) A high frequency of apolipoprotein E4 isoprotein in Japanese patients with late-onset nonfamilial Alzheimer's disease. Neurosci Lett 163: 166-168.

7. Schmechel DE, Saunders AM, Strittmatter WJ, Crain BJ, Hulette CM, et al. (1993) Increased amyloid beta-peptide deposition in cerebral cortex as a consequence of apolipoprotein $\mathrm{E}$ genotype in late-onset Alzheimer disease. Proc Natl Acad Sci U S A 90: 9649-9653.

8. Saunders AM, Schmader K, Breitner JC, Benson MD, Brown WT, et al. (1993) Apolipoprotein $\mathrm{E}$ epsilon 4 allele distributions in late-onset Alzheimer's disease and in other amyloid-forming diseases. Lancet 342: 710-711.

9. Lahoz CE, Schaefer J, Cupples LA, Wilson PW, Levy D, et al. (2001)
Apolipoprotein E genotype and cardiovascular disease in the Framingham Heart Study. Atherosclerosis 154: 529-537.

10. Mahley RW, Rall SC Jr (2000) Apolipoprotein E: far more than a lipid transport protein. Annu Rev Genomics Hum Genet 1: 507-537.

11. Poirier J, Hess M, May PC, Finch CE (1991) Astrocytic apolipoprotein E mRNA and GFAP mRNA in hippocampus after entorhinal cortex lesioning. Brain Res Mol Brain Res 11: 97-106.

12. Boyles JK, Pitas RE, Wilson E, Mahley RW, Taylor JM (1985) Apolipoprotein $\mathrm{E}$ associated with astrocytic glia of the central nervous system and with nonmyelinating glia of the peripheral nervous system. J Clin Invest 76: 15011513.

13. Bu G (2009) Apolipoprotein E and its receptors in Alzheimer's disease pathways, pathogenesis and therapy. Nat Rev Neurosci 10: 333-344.

14. Xu PT, Schmechel D, Qiu HL, Herbstreith M, Rothrock-Christian T, et al. (1999) Sialylated human apolipoprotein E (apoEs) is preferentially associated with neuron-enriched cultures from APOE transgenic mice. Neurobiol Dis 6: 63-75.

15. Beffert U, Poirier J (1996) Apolipoprotein E, plaques, tangles and cholinergic dysfunction in Alzheimer's disease. Ann N Y Acad Sci 777: 166-174.

16. Metzger RE, LaDu MJ, Pan JB, Getz GS, Frail DE, et al. (1996) Neurons of the human frontal cortex display apolipoprotein $E$ immunoreactivity: implications for Alzheimer's disease. J Neuropathol Exp Neurol 55: 372-380.

17. Han SH, Einstein G, Weisgraber KH, Strittmatter WJ, Saunders AM, et al. (1994) Apolipoprotein $E$ is localized to the cytoplasm of human cortical neurons: a light and electron microscopic study. J Neuropathol Exp Neurol 53: 535-544.

18. Huang $Y$ (2006) Molecular and cellular mechanisms of apolipoprotein E4 neurotoxicity and potential therapeutic strategies. Curr Opin Drug Discov Devel 9: 627-641.

19. Huang Y, Weisgraber KH, Mucke L, Mahley RW (2004) Apolipoprotein E: diversity of cellular origins, structural and biophysical properties, and effects in Alzheimer's disease. J Mol Neurosci 23: 189-204.

20. Murphy C, Bacon AW, Bondi MW, Salmon DP (1998) Apolipoprotein E status is associated with odor identification deficits in nondemented older persons. Ann N Y Acad Sci 855: 744-750.

21. Doty RL (2009) The olfactory system and its disorders. Semin Neurol 29: 74-81.

22. Graves AB, Bowen JD, Rajaram L, McCormick WC, McCurry SM, et al (1999) Impaired olfaction as a marker for cognitive decline: interaction with apolipoprotein E epsilon4 status. Neurology 53: 1480-1487.

23. Chess A, Simon I, Cedar H, Axel R (1994) Allelic inactivation regulates olfactory receptor gene expression. Cell 78: 823-834.

24. Mombaerts P, Wang F, Dulac C, Chao SK, Nemes A, et al. (1996) Visualizing an olfactory sensory map. Cell 87: 675-686.

25. Kato A, Reisert J, Ihara S, Yoshikawa K, Touhara K (2014) Evaluation of the role of $g$ protein-coupled receptor kinase 3 in desensitization of mouse odoran receptors in a Mammalian cell line and in olfactory sensory neurons. Chem Senses 39: 771-780.

26. Yoshikawa K, Touhara K (2009) Myr-Ric-8A enhances G(alpha15)-mediated $\mathrm{Ca} 2+$ response of vertebrate olfactory receptors. Chem Senses 34: 15-23.

27. Bhandawat V, Reisert J, Yau KW (2005) Elementary response of olfactory receptor neurons to odorants. Science 308: 1931-1934.

28. Araneda RC, Kini AD, Firestein S (2000) The molecular receptive range of an odorant receptor. Nat Neurosci 3: 1248-1255.

29. Verhagen JV, Wesson DW, Netoff TI, White JA, Wachowiak M (2007) Sniffing controls an adaptive filter of sensory input to the olfactory bulb. Nat Neurosci 10: 631-639.

30. Buonviso N, Amat C, Litaudon P (2006) Respiratory modulation of olfactory neurons in the rodent brain. Chem Senses 31: 145-154.

31. Mozell MM (1964) Olfactory Discrimination: Electrophysiological Spatiotemporal Basis. Science 143: 1336-1337.

32. Spors H, Wachowiak M, Cohen LB, Friedrich RW (2006) Temporal dynamics and latency patterns of receptor neuron input to the olfactory bulb. J Neurosci 26: $1247-1259$.

33. Johnson BA, Farahbod H, Xu Z, Saber S, Leon M (2004) Local and global 
Citation: Misiak MM, Hipolito MMS, Ressom HW, Obisesan TO, Manaye KF, et al. (2017) Apo E4 Alleles and Impaired Olfaction as Predictors of Alzheimer's Disease. Clin Exp Psychol 3: 169. doi: 10.4172/2471-2701.1000169

chemotopic organization: general features of the glomerular representations of aliphatic odorants differing in carbon number. J Comp Neurol 480: 234-249.

34. UchidaN, Takahashi YK, Tanifuji M, Mori K (2000) Odor maps in the mammalian olfactory bulb: domain organization and odorant structural features. Nat Neurosci 3: 1035-1043.

35. Sharp FR, Kauer JS, Shepherd GM (1975) Local sites of activity-related glucose metabolism in rat olfactory bulb during olfactory stimulation. Brain Res 98: $596-600$

36. Wachowiak M, Shipley MT (2006) Coding and synaptic processing of sensory information in the glomerular layer of the olfactory bulb. Semin Cell Dev Biol 17: 411-423.

37. Hayar A, Karnup S, Ennis M, Shipley MT (2004) External tufted cells: a major excitatory element that coordinates glomerular activity. J Neurosci 24: 66766685.

38. Aungst JL, Heyward PM, Puche AC, Karnup SV, Hayar A, et al. (2003) Centresurround inhibition among olfactory bulb glomeruli. Nature 426: 623-629.

39. Tan J, Savigner A, Ma, M, Luo M (2010) Odor information processing by the olfactory bulb analyzed in gene-targeted mice. Neuron 65: 912-926.

40. Brill MS, Ninkovic J, Winpenny E, Hodge RD, Ozen I, et al. (2009) Adult generation of glutamatergic olfactory bulb interneurons. Nat Neurosci 12: 15241533.

41. Han YG, Spassky N, Romaguera-Ros M, Garcia-Verdugo JM, Aguilar A, et al. (2008) Hedgehog signaling and primary cilia are required for the formation of adult neural stem cells. Nat Neurosci 11: 277-284.

42. Imayoshi I, Sakamoto M, Ohtsuka T, Takao K, Miyakawa T, et al. (2008) Roles of continuous neurogenesis in the structural and functional integrity of the adult forebrain. Nat Neurosci 11: 1153-1161.

43. Lois C, Alvarez-Buylla A (1994) Long-distance neuronal migration in the adult mammalian brain. Science 264: 1145-1148.

44. Linster C, Fontanini A (2014) Functional neuromodulation of chemosensation in vertebrates. Curr Opin Neurobiol 29: 82-87.

45. Kay RB, Meyer EA, Illig KR, Brunjes PC (2011) Spatial distribution of neura activity in the anterior olfactory nucleus evoked by odor and electrical stimulation. J Comp Neurol 519: 277-289.

46. Kikuta S, Sato K, Kashiwadani H, Tsunoda K, Yamasoba T, et al. (2010) From the Cover: Neurons in the anterior olfactory nucleus pars externa detect right or left localization of odor sources. Proc Natl Acad Sci U S A 107: 12363-12368.

47. Wilson DA, Sullivan RM (2011) Cortical processing of odor objects. Neuron 72: 506-19.

48. Gottfried JA (2010) Central mechanisms of odour object perception. Nat Rev Neurosci 11: 628-641.

49. Barkai E, Saar D (2001) Cellular correlates of olfactory learning in the rat piriform cortex. Rev Neurosci 12: 111-120.

50. Schoenbaum G, Eichenbaum H (1995a) Information coding in the rodent prefrontal cortex. I. Single-neuron activity in orbitofrontal cortex compared with that in pyriform cortex. J Neurophysiol 74: 733-750.

51. Schoenbaum G, Eichenbaum H (1995c) Information coding in the roden prefrontal cortex. II. Ensemble activity in orbitofrontal cortex. J Neurophysio 74: 751-762.

52. Gadziola MA, Wesson DW (2016) The Neural Representation of Goal-Directed Actions and Outcomes in the Ventral Striatum's Olfactory Tubercle. J Neurosci 36: $548-560$

53. Gadziola MA, Tylicki KA, Christian DL, Wesson DW (2015) The olfactory tubercle encodes odor valence in behaving mice. J Neurosci 35: 4515-4527.

54. Doty RL, Philip S, Reddy K, Kerr KL (2003) Influences of antihypertensive and antihyperlipidemic drugs on the senses of taste and smell: a review. J Hypertens 21: 1805-1813.

55. Gomez-Isla T, Price JL, McKeel DW Jr, Morris JC, Growdon JH (1996) Profound loss of layer II entorhinal cortex neurons occurs in very mild Alzheimer's disease. J Neurosci 16: 4491-4500

56. Kareken DA, Mosnik DM, Doty RL Dzemidzic M. Hutchins GD (2003) Functional anatomy of human odor sensation, discrimination, and identification in health and aging. Neuropsychology 17: 482-495.
57. Lotsch J, Reichmann H, Hummel T (2008) Different odor tests contribute differently to the evaluation of olfactory loss. Chem Senses 33: 17-21.

58. Kobal G, Hummel T, Sekinger B, Barz S, Roscher S, et al. (1996) "Sniffin" sticks": screening of olfactory performance. Rhinology 34: 222-226.

59. Doty RL, Shaman P, Applebaum SL, Giberson R, Siksorski L, et al. (1984) Smell identification ability: changes with age. Science 226: 1441-1443.

60. Frasnelli J, Hummel T (2005) Olfactory dysfunction and daily life. Eur Arch Otorhinolaryngol 262: 231-235.

61. Plailly J, d'Amato T, Saoud M, Royet JP (2006) Left temporo-limbic and orbita dysfunction in schizophrenia during odor familiarity and hedonicity judgments. Neuroimage 29: 302-313.

62. Joussain P, Thevenet M, Rouby C, Bensafi M (2013) Effect of aging on hedonic appreciation of pleasant and unpleasant odors. PLoS One 8: e61376.

63. Suzuki Y, Critchley HD, Rowe A, Howlin P, Murphy DG (2003) Impaired olfactory identification in Asperger's syndrome. J Neuropsychiatry Clin Neurosci 15: 105107

64. Zatorre RJ, Jones-Gotman M (1991) Human olfactory discrimination after unilateral frontal or temporal lobectomy. Brain 114( Pt 1A): 71-84.

65. Tanabe T, lino M, Takagi SF (1975) Discrimination of odors in olfactory bulb, pyriform-amygdaloid areas, and orbitofrontal cortex of the monkey. $J$ Neurophysiol 38: 1284-1296

66. Jackman AH, Doty RL (2005) Utility of a three-item smell identification test in detecting olfactory dysfunction. Laryngoscope 115: 2209-2212.

67. Doty RL, Marcus A, Lee WW (1996) Development of the 12-item Cross-Cultura Smell Identification Test (CC-SIT). Laryngoscope 106: 353-356.

68. Simmen D, Briner HR, Hess K (1999) [Screening of olfaction with smell diskettes]. Laryngorhinootologie 78: 125-130.

69. Nordin S, Bramerson A, Liden E, Bende M (1998) The Scandinavian OdorIdentification Test: development, reliability, validity and normative data. Acta Otolaryngol 118: 226-234.

70. Toyota B, Kitamura T, Takagi SF (1978) Olfactory Disorders-Olfactometry and Therapy. Igaku-Shoin, Tokyo.

71. Hummel T, Sekinger B, Wolf SR, Pauli E, Kobal G (1997) 'Sniffin' sticks': olfactory performance assessed by the combined testing of odor identification odor discrimination and olfactory threshold. Chem Senses 22: 39-52.

72. Deems DA, Doty RL (1987) Age-related changes in the phenyl ethyl alcoho odor detection threshold. Trans Pa Acad Ophthalmol Otolaryngol 39: 646-650.

73. Guarneros M, Ortiz-Romo N, Alcaraz-Zubeldia M, Drucker-Colin R, Hudson $R$ (2013) Nonoccupational environmental exposure to manganese is linked to deficits in peripheral and central olfactory function. Chem Senses 38: 783-791.

74. Doty RL (2000) The Smell Threshold Test ${ }^{\mathrm{TM}}$ administration manual. Sensonics Inc, Haddon Heights, $\mathrm{NJ}$

75. Sulmont C, Issanchou S, Köster EP (2002) Selection of odorants for memory tests on the basis of familiarity, perceived complexity, pleasantness, similarity and identification. Chem Senses 27: 307-317.

76. Lehrner JP, Brücke T, Dal-Bianco P, Gatterer G, Kryspin-Exner I (1997) Olfactory functions in Parkinson's disease and Alzheimer's disease. Chem Senses 22: 105-110.

77. Landis BN, Negoias S, Friedrich H (2016) Chemosensory Event Related Potentials. Epileptologie 33: 189-196.

78. Miao X, Yang L, Gu H, Ren Y, Chen G, et al. (2015) Evaluation of post-traumatic anosmia with MRI and chemosensory ERPs. Eur Arch Otorhinolaryngol 272 1945-1953.

79. Barresi M, Ciurleo R, Giacoppo S, Foti Cuzzola V, Celi D, et al (2012) Evaluation of olfactory dysfunction in neurodegenerative diseases. $\mathrm{J}$ Neurol Sci 323: 16-24

80. Rombaux P, Mouraux A, Bertrand B, Guerit JM, Hummel T (2006) Assessment of olfactory and trigeminal function using chemosensory event-related potentials. Neurophysiol Clin 36: 53-62.

81. Kappenman ES, Luck SJ (2010) The effects of electrode impedance on data quality and statistical significance in ERP recordings. Psychophysiology 47 888-904. 
Citation: Misiak MM, Hipolito MMS, Ressom HW, Obisesan TO, Manaye KF, et al. (2017) Apo E4 Alleles and Impaired Olfaction as Predictors of Alzheimer's Disease. Clin Exp Psychol 3: 169. doi: 10.4172/2471-2701.1000169

Page 13 of 13

82. Murphy C, Solomon ES, Haase L, Wang M, Morgan CD (2009) Olfaction in aging and Alzheimer's disease: event-related potentials to a cross-modal odorrecognition memory task discriminate ApoE epsilon4+ and ApoE epsilon 4individuals. Ann N Y Acad Sci 1170: 647-657.

83. Calhoun-Haney R, Murphy C (2005) Apolipoprotein epsilon4 is associated with more rapid decline in odor identification than in odor threshold or Dementia Rating Scale scores. Brain Cogn 58: 178-182.

84. Gilber PE, Murphy C (2004) The effect of the ApoE epsilon4 allele on recognition memory for olfactory and visual stimuli in patients with pathologically confirmed Alzheimer's disease, probable Alzheimer's disease, and healthy elderly controls. J Clin Exp Neuropsychol 26: 779-794.

85. Wang QS, Tian L, Huang YL, Qin S, He LQ, et al. (2002) Olfactory identification and apolipoprotein E epsilon 4 allele in mild cognitive impairment. Brain Res 951: 77-81.

86. Bacon AW, Bondi MW, Salmon DP, Murphy C (1998) Very early changes in olfactory functioning due to Alzheimer's disease and the role of apolipoprotein E in olfaction. Ann N Y Acad Sci 855: 723-731.

87. Sundermann EE, Gilbert PE, Murphy C (2008) The effect of hormone therapy on olfactory sensitivity is dependent on apolipoprotein $E$ genotype. Horm Behav 54: 528-533.

88. Sundermann EE, Gilbert PE, Murphy C (2007) Apolipoprotein E epsilon4 genotype and gender: effects on memory. Am J Geriatr Psychiatry 15:69-78.

89. Kjelvik G, Saltvedt I, White LR, Stenumgard P, Sletvold O, et al. (2014) The brain structural and cognitive basis of odor identification deficits in mild cognitive impairment and Alzheimer's disease. BMC Neurol 14: 168.

90. Handley OJ, Morrison CM, Miles C, Bayer AJ (2006) ApoE gene and familial risk of Alzheimer's disease as predictors of odour identification in older adults. Neurobiol Aging 27: 1425-1430.

91. Oleson S, Murphy C (2015) Olfactory Dysfunction in ApoE varepsilon4/4 Homozygotes with Alzheimer's Disease. J Alzheimers Dis 46: 791-803.

92. Zigman WB, Devenny DA, Krinsky-McHale SJ, Jenkins ED, Urv TK, et al. (2008) Alzheimer's Disease in Adults with Down Syndrome. Int Rev Res Ment Retard 36: 103-145.

93. Sliger M, Lander T, Murphy C (2004) Effects of the ApoE epsilon4 allele on olfactory function in Down syndrome. J Alzheimers Dis 6: 397-402.

94. Wetter S, Murphy C (2001) Apolipoprotein E epsilon4 positive individuals demonstrate delayed olfactory event-related potentials. Neurobiol Aging 22 : 439-447.

95. Murphy C, Morgan CD, Geisler MW, Wetter S, Covington J, et al. (2000) Olfactory event-related potentials and aging: normative data. Int J Psychophysiol 36: 133-145.

96. Covington JW, Geisler MW, Polich J, Murphy C (1999) Normal aging and odor intensity effects on the olfactory event-related potential. Int J Psychophysio 32: $205-214$.

97. Corby K, Morgan CD, Murphy C (2012) Abnormal event-related potentials in young and middle-aged adults with the ApoE epsilon4 allele. Int J Psychophysiol 83: $276-281$.

98. Kowalewski J, Murphy C (2012) Olfactory ERPs in an odor/visual congruency task differentiate ApoE epsilon4 carriers from non-carriers. Brain Res 1442:
$5-65$.

99. Morgan CD, Murphy C (2012) Individuals at risk for Alzheimer's disease show differential patterns of ERP brain activation during odor identification. Behav Brain Funct 8: 37.

100. Peng KY, Matthews PM, Levy E, Wilson DA (2017) Apolipoprotein E4 causes early olfactory network abnormalities and short-term olfactory memory impairments. Neuroscience 343: 364-371.

101. Nathan BP, Yost J, Litherland MR, Struble RG, Switzer PV (2004) Olfactory function in apoE knockout mice. Behav Brain Res 150: 1-7.

102. Nathan BP, Nannapaneni S, Gairhe S, Nwosu I, Struble RG (2007) The distribution of apolipoprotein $\mathrm{E}$ in mouse olfactory epithelium. Brain Res 1137 78-83.

103. Nathan BP, Nisar R, Short J, Randall S, Grissom E, et al. (2005) Delayed olfactory nerve regeneration in ApoE-deficient mice. Brain Res 1041: 87-94.

104. Hussain A, Luong M, Pooley A, Nathan BP (2013) Isoform-specific effects of apoE on neurite outgrowth in olfactory epithelium culture. J Biomed Sci 20: 49

105. Nwosu I, Gairhe S, Struble RG, Nathan BP (2008) Impact of apoE deficiency during synaptic remodeling in the mouse olfactory bulb. Neurosci Lett 441 : 282-285.

106. Nathan BP, Gairhe S, Nwosu I, Clark S, Struble RG (2010a) Reconstitution of the olfactory epithelium following injury in apoE-deficient mice. Exp Neurol 226: $40-46$.

107. Chiba S, Suzuki M, Yamanouchi K, Nishihara M (2007) Involvement of granulin in estrogen-induced neurogenesis in the adult rat hippocampus. J Reprod Dev 53: $297-307$.

108. Morris DC, Zhang ZG, Wang Y, Zhang RL Gregg S, et al. (2007) Wnt expression in the adult rat subventricular zone after stroke. Neurosci Lett 418: 170-174.

109. Caruso A, Motolese M, lacovelli L, Caraci F, Copani A, et al. (2006) Inhibition of the canonical Wnt signaling pathway by apolipoprotein E4 in PC12 cells. J Neurochem 98: 364-371.

110. Nathan BP, Tonsor M, Struble RG (2012) Long-term effects of estradio replacement in the olfactory system. Exp Neurol 237: 1-7.

111. Nathan BP, Tonsor M, Struble RG (2010c) Acute responses to estradio replacement in the olfactory system of apoE-deficient and wild-type mice. Brain Res 1343: 66-74

112. Cheng X, McAsey ME, Li M, Randall S, Cady C, et al. (2007) Estradio replacement increases the low-density lipoprotein receptor related protein (LRP) in the mouse brain. Neurosci Lett 417: 50-54

113. McAsey ME, Cady C, Jackson LM, Li M, Randall S, et al. (2006) Time course of response to estradiol replacement in ovariectomized mice: brain apolipoprotein E and synaptophysin transiently increase and glial fibrillary acidic protein is suppressed. Exp Neurol 197: 197-205.

114. Richey PL, Siedlak SL, Smith MA, Perry G (1995) Apolipoprotein E interaction with the neurofibrillary tangles and senile plaques in Alzheimer disease: implications for disease pathogenesis. Biochem Biophys Res Commun 208: 657-663

115. Carter DB (2005) The interaction of amyloid-beta with ApoE. Subcell Biochem 38: $255-272$. 